\title{
On a free boundary barotropic model
}

by

\section{P.-L. LIONS}

CEREMADE-URA CNRS 749 Université Paris Dauphine, place de Lattre de Tassigny, 75775 Paris cedex 16. France

and

\section{N. MASMOUDI*}

DMI, École Normale Supérieure, 45 rue d’Ulm, 75005 Paris, France

ABSTRACT. - We prove stability (or compactness) and existence results for a free boundary model of a barotropic compressible fluid. Then, we construct weak solutions as asymptotic limits of the isentropic compressible Navier-Stokes equations as $\gamma$ goes to $\infty$.

(c) 1999 L'Association Publications de l'Institut Henri Poincaré. Published by Elsevier B.V. All rights reserved

RÉSUMÉ. - Nous montrons l'existence de solutions faibles globales pour un modèle de fluide compressible barotrope. Ces solutions sont construites à partir d'une limite asymptotique des solutions de Navier-Stokes isentropique compressible.

(C) 1999 L'Association Publications de l'Institut Henri Poincaré. Published by Elsevier B.V. All rights reserved

\section{INTRODUCTION}

We consider the following system of equations, written in $(0, \infty) \times \Omega$, where $\Omega=\mathbb{R}^{\mathrm{N}}$ or $\Omega=\mathbf{T}^{\mathrm{N}}$,

(1) $\frac{\partial \rho}{\partial t}+\operatorname{div}(\rho u)=0$ in $(0, T) \times \Omega, 0 \leq \rho \leq 1$ in $(0, T) \times \Omega$,

* CEREMADE-URA CNRS 749 Université Paris Dauphine, place de Lattre de Tassigny, 75775 Paris cedex 16, France.

AMS Classifications: $76 \mathrm{~N} 10,76 \mathrm{~T} 05$.

Annales de I'Institut Henri Poincaré - Analyse non linéaire - 0294-1449

Vol. 16/99/03/

(C) 1999 L'Association Publications de l'Institut Henri Poincaré. Published by Elsevier B.V. All rights reserved 
(2) $\frac{\partial \rho u}{\partial t}+\operatorname{div}(\rho u \otimes u)-\mu \Delta u-\xi \nabla \operatorname{div} u+\nabla \pi=\rho f$ in $(0, T) \times \Omega$,

$$
\begin{gathered}
\operatorname{div} u=0 \text { a.e. on }\{\rho=1\} \\
\pi=p(\rho) \text { a.e. on }\{\rho<1\}, \pi \geq p(1) \text { a.e. on }\{\rho=1\}
\end{gathered}
$$

where $T \in(0, \infty), \mu>0, \mu+\xi>0, p(\rho)$ is a nondecreasing positive continuous function and $f=f(t, x)$ is a given function corresponding to the volumic force terms (for instance we can assume that $f \in L^{1}\left(0, T ; L^{2}\right)^{N}$ ).

The unknowns $(\rho, u, \pi)$ correspond respectively to the density of the fluid which is a nonnegative function, the velocity which is a vector-valued function in $\mathbb{R}^{\mathrm{N}}$ and the pressure. The system must be complemented with initial conditions, namely

$$
\left.\rho u\right|_{t=0}=m^{0},\left.\rho\right|_{t=0}=\rho^{0} .
$$

where $1 \geq \rho^{0} \geq 0$ a.e. , $\rho^{0} \in L^{1}(\Omega), m^{0} \in L^{2}(\Omega), m^{0}=0$ a.e. on $\left\{\rho^{0}=0\right\}, \rho^{0} \neq 0$, and $\rho^{0}\left|u^{0}\right|^{2} \in L^{1}$, denoting by $u^{0}=\frac{m^{0}}{\rho^{0}}$ on $\left\{\rho^{0}>0\right\}$, $u^{0}=0$ on $\left\{\rho^{0}=0\right\}$. In the case of $\mathbf{T}^{\mathrm{N}}$, we also impose that $f \rho^{0}=M<1$, otherwise the above system reduces formally to the incompressible NavierStokes equations in which case we do not know whether the (hydrostatic) pressure is bounded from below.

One of the motivations to study this free boundary problems is the study of fluids with imbedded domains (large bubbles) filled with gaz : standard models involve a threshold on the pressure beyond which one has the incompressible Navier-Stokes equations for the fluid and below which one has a compressible model for the gaz. Another motivation is the possibility to study a compressible-like system which includes the incompressible case as particular case $(\rho \equiv 1)$.

Now we are going to define precisely the weak solutions (solutions à la Leray) we will use. We look for solutions satisfying

$$
\begin{gathered}
\rho \in L^{\infty}\left(0, T ; L^{\infty} \cap L^{1}(\Omega)\right) \cap C\left(0, T ; L^{p}\right) \text { for any } 1 \leq p<\infty \\
\nabla u \in L^{2}\left(0, T, L^{2}\right) \text { and } u \in L^{2}\left(0, T ; H^{1}(B)\right),
\end{gathered}
$$

where $B=\mathrm{T}^{\mathrm{N}}$ if $\Omega=\mathbf{T}^{\mathrm{N}}$ and $B$ is any ball in $\mathbb{R}^{\mathrm{N}}$ if $\Omega=\mathbb{R}^{N}$, in this second case we also impose that $u \in L^{2}\left(0, T, L^{2 N / N-2}\left(\mathbb{R}^{N}\right)\right)$, if in addition $N \geq 3$. 
We also require

$$
\rho|u|^{2} \in L^{\infty}\left(0, \infty ; L^{1}\right) \text { and } \rho u \in L^{\infty}\left(0, \infty ; L^{2}\right)
$$

Finally we impose that

$$
\pi \in \mathcal{M}((0, T) \times \Omega),
$$

where $\mathcal{M}((0, T) \times \Omega)$ is the space of bounded measures on $(0, T) \times \Omega$.

Next, equations (1), (2) must be satisfied in the distributional sense. This can be written using a weak formulation, namely we require that the following identities hold for all $\phi \in C^{\infty}([0, \infty) \times \Omega)$ and for all $\Phi \in C^{\infty}([0, \infty) \times \Omega)^{N}$ compactly supported in $[0, \infty) \times \Omega$ (i.e. vanishing identically for $t$ large enough)

$$
-\int_{0}^{\infty} d t \int_{\Omega} \rho \partial_{t} \phi-\int_{\Omega} \rho^{0} \phi(0)-\int_{0}^{\infty} d t \int_{\Omega} \rho u . \nabla \phi=0,
$$

$$
\begin{aligned}
& -\int_{0}^{\infty} d t \int_{\Omega} \rho u . \partial_{t} \Phi-\int_{\Omega} m^{0} . \Phi(0)-\int_{0}^{\infty} d t \int_{\Omega} \rho(u . \nabla \Phi) . u+ \\
& +\int_{0}^{\infty} d t\left\{\int_{\Omega} \mu D u . D \Phi+\xi \operatorname{div} u \operatorname{div} \Phi\right\}-\pi \operatorname{div} \Phi=\int_{0}^{\infty} d t \int_{\Omega} \rho f . \Phi .
\end{aligned}
$$

We want to point out (and we will come back to this issue later on) that the weak formulation (10) for $\rho$ contains the initial condition $\rho(0)=\rho^{0}$ since $\rho$ is assumed to be continous in time with values in $L^{2}$. However (11) does not yield that $\rho u(0)=m^{0}$. In fact $\pi$ is a mesure and all we can deduce in terms of continuity concerns the divergence-free part of $\rho u$, namely $P(\rho u)$, where $P(v)=v-\nabla(\Delta)^{-1} \nabla . v$. Hence if $\Phi$ is divergence-free $\operatorname{div}(\Phi)=0$ then (11) becomes

$$
\left\{\begin{array}{l}
-\int_{0}^{\infty} d t \int_{\Omega} \rho u \cdot \partial_{t} \Phi-\int_{\Omega} m^{0} \cdot \Phi(0)-\int_{0}^{\infty} d t \int_{\Omega} \rho(u \cdot \nabla \Phi) \cdot u+ \\
+\int_{0}^{\infty} d t \int_{\Omega} \mu D u . D \Phi=\int_{0}^{\infty} d t \int_{\Omega} \rho f . \Phi
\end{array}\right.
$$

which yields that $P(\rho u)(0)=P\left(m^{0}\right)$.

On the other hand equation (3) and the condition $0 \leq \rho \leq 1$ must be understood in the sense of almost everywhere defined functions. 
Notice then that condition (4) does not make sense since $\pi$ is not assumed to be a function (defined almost every where). However this condition can be rewritten as follows

$$
\rho(\pi-p(\rho))=\pi-p(\rho) \geq 0
$$

We will show that this condition makes sense under the above requirements and equation (4) should be understood in the sense of (13). In fact we will show the following regularity theorem

THEOREM 1.1. - If $(\rho, u, \pi)$ satisfy (I), (2) and the above requirements hold then condition (13) makes sense and we have

$$
\begin{gathered}
\pi \in \mathcal{M}\left(0, T ; L^{1}(\Omega)\right), \\
\rho u \in B V\left([0, \infty) ; L^{2}(\Omega)-w\right), \\
P(\rho u) \in C\left([0, \infty) ; L^{2}(\Omega)-w\right) .
\end{gathered}
$$

In this result, $B V\left([0, \infty) ; L^{2}-w\right)$ and $C\left([0, \infty) ; L^{2}-w\right)$ are respectively the space of bounded variation and continous functions on $[0, \infty)$ with value in a bounded set of $L^{2}(\Omega)$ equipped with the weak topology.

Now we are able to state our main existence result. As is customary when dealing with global weak solutions of partial differential equations (and due to the weak convergences in the approximating systems) the global weak solution we are going to construct will satisfy in addition the following energy inequalities

$$
\begin{gathered}
E(t)+\int_{0}^{t} D(s) d s \leq E^{0}+\int_{0}^{t} \int_{\Omega} \rho u . f \text { a.e. } t . \\
\frac{d E}{d t}+D \leq \int_{\Omega} \rho u . f \text { in } \mathcal{D}^{\prime}(0, \infty)
\end{gathered}
$$

where $E(t)=\int_{\Omega} \frac{1}{2} \rho|u|^{2}(t), D(t)=\int_{\Omega} \mu|D u|^{2}(t)+\xi(\text { div } u)^{2}(t)$ and $E^{0}=\int_{\Omega} \frac{1}{2} \rho^{0}\left|u^{0}\right|^{2}$. Moreover, $\pi$ will be bounded in $\mathcal{M}((0, T) \times \Omega)$ by a constant depending on the initial data and on $f$.

$$
\|\pi\|_{\mathcal{M}((0, T) \times \Omega)} \leq C\left(\rho^{0}, m^{0}, f\right)
$$


THEOREM 1.2. - Besides the conditions on the initial data already given above we assume that $p(\rho)=0$ and that $f$ satisfies the following condition

$$
\left\{\begin{array}{l}
f \in L^{1}\left(0, T ; L^{2}(\Omega)\right)+L^{2}\left(0, T ; L^{r}(\Omega)\right) \text { if } \Omega=\mathbf{T}^{2} \\
f \in L^{1}\left(0, T ; L^{2}+L^{\infty}(\Omega)\right)+L^{2}\left(0, T ; L^{r}(B)\right) \text { if } \Omega=\mathbb{R}^{2} \\
f \in L^{1}\left(0, T ; L^{2}+L^{\infty}(\Omega)\right)+L^{2}\left(0, T ; L^{\frac{2 N}{N+2}}(\Omega)\right) \text { if } N \geq 3
\end{array}\right.
$$

where $1<r \leq 2$ and $B$ is a bounded ball in $\mathbb{R}^{2}$, then there exists a solution $(\rho, u, \pi)$ for the system (1) - (4) satisfying the above requirement and the energy inequality.

In the next sections, we shall give precise conditions on $p(\rho)$, and state more general results. Besides, the requirements on $\rho$ can be weakened (in the case of $\mathbb{R}^{\mathrm{N}}$ ), in fact we can take a fluid of infinite mass. For instance we can impose the following condition

$$
\rho^{0}-\bar{\rho} \in L^{\infty}\left(0, \infty ; L^{1}\left(\mathbb{R}^{\mathrm{n}}\right)\right)
$$

for some constant $\bar{\rho}$, such that $0<\bar{\rho}<1$ (or more generally for some reference function $\bar{\rho}$ in $\mathbb{R}^{\mathrm{N}}$ satisfying $0 \leq \bar{\rho} \leq 1$ ), we will come back to this issue in details in section 5 .

A priori estimates will be derived in section 2 , In section 3 we study the compactness of sequences of solutions satisfying the requirement above, then the existence results will be proved in section 4, using the convergence of solutions of the compressible isentropique Navier-Stokes equations as $\gamma$ goes to the infinity (where the pressure is given by $p(\rho)=\rho^{\gamma}$ ). Finally in section 6, we give two convergence results to the incompressible Navier-Stokes system.

\section{A PRIORI ESTIMATES}

We are going to show here that the notion of weak solutions we have defined above is a natural one by showing some a priori bounds. First, we can notice that the conditions (1) and (3) are compatible, in fact we have the following lemma

Lemma 2.1. - Let $u \in L^{2}\left(0, T ; H_{l o c}^{1}\right)$ and $\rho \in L_{\text {loc }}^{2}$ satisfying

$$
\frac{\partial \rho}{\partial t}+\operatorname{div}(\rho u)=0 \text { in }(0, T) \times \Omega, \quad \rho(0)=\rho^{0}
$$


then the following two assertions are equivalent

1. div $u=0$ a.e. on $\{\rho \geq 1\}$ and $0 \leq \rho^{0} \leq 1$,

2. $0 \leq \rho \leq 1$.

Proof. - Let us begin by the first implication $(1) \rightarrow 2$ ) ), using the regularization lemma stated in [7] (lemma 2.3 p 43), we get

$$
\frac{\partial \beta(\rho)}{\partial t}+\operatorname{div}(\beta(\rho) u)=\left(\beta(\rho)-\rho \beta^{\prime}(\rho)\right) \operatorname{div} u
$$

for any $C^{1}$ function $\beta$ from $\mathbb{R}$ to $\mathbb{R}$ such that $|\beta(t)| \leq C+C t$. Next let $\beta$ be defined by

$$
\left\{\begin{array}{lll}
\beta(t)=0 & \text { if } & t \leq 0 \\
\beta(t)=t & \text { if } & 0 \leq t \leq 1, \\
\beta(t)=1 & \text { if } & 1 \leq t .
\end{array}\right.
$$

then we get

$$
\frac{\partial \beta(\rho)}{\partial t}+\operatorname{div}(\beta(\rho) u)=1_{\{\rho \geq 1\}} \operatorname{div}(u)=0,
$$

in fact taking any sequence of $C^{1}$ functions $\beta_{n}$ such that $\beta_{n}(l)=\beta(l)$ on $]-\infty,-\frac{1}{n}[\cup] \frac{1}{n}, 1-\frac{1}{n}[\cup] 1+\frac{1}{n},+\infty\left[\right.$ and $\left|\beta^{\prime}(t)\right| \leq C$ uniformly in $n$, we can rewrite (21) with $\beta$ replaced by $\beta_{n}$, then $\beta_{n}(\rho)$ converges pointwise and in $L^{2}$ to $\beta(\rho)$. Moreover $\left(\beta_{n}(\rho)-\rho \beta_{n}^{\prime}(\rho)\right)$ div $u$ (which is bounded in $L_{\text {loc }}^{2}$ ) converges pointwise to 0 , and we can then recover (23). In addition we have $\beta(\rho)(t=0)=\rho^{0}$; and setting $d=\beta(\rho)-\rho$, we see that $d$ solves the same equation as $\rho$ and $\beta(\rho)$ and that $d(0)=0$. Applying the regularization lemma another time, we see that $|d|$ also solves the same equation and since $\int_{\Omega}|d|(t)=\int_{\Omega}|d|(0)$ for any $t$, we get that $d=0$ and hence $\beta(\rho)=\rho$ which yields 2 ).

Now, we turn out to the proof of the second implication. Since $0 \leq \rho \leq 1$, we see that $\rho$ is bounded and then (21) holds for any $C^{1}$ function $\beta$. Writing it for $\beta(\rho)=\rho^{k}$, for any integer $\mathbf{k}$, we get

$$
\frac{\partial \rho^{k}}{\partial t}+\operatorname{div}\left(\rho^{k} u\right)=(1-k) \rho^{k} \operatorname{div} u
$$

Since $0 \leq \rho^{k} \leq 1$, we see that $\partial_{t} \rho^{k}$ is bounded in $W^{-1, \infty}((0, T) \times \Omega)$, in addition $\operatorname{div}\left(\rho^{k} u\right)$ is bounded in $L^{\infty}\left(0, T ; I I_{l o c}^{-1}(\Omega)\right)$, for $\left|\rho^{k} u\right| \leq|\rho u| \epsilon$ $L^{\infty}\left(L_{l o c}^{2}\right)$. This yields that $k \rho^{k} \operatorname{div} u$ is a bounded distribution (in $H_{l o c}^{-1}$ for instance). Letting $k$ go to the infinity, we get

$$
\rho^{k} \operatorname{div} u \underset{k}{\rightarrow} 0 \text { in } \mathcal{D}^{\prime} \text {. }
$$


Besides, we have

$$
\rho^{k} \rightarrow 1_{\{\rho=1\}} \quad \text { a.e }
$$

so we get

$$
\rho^{k} \operatorname{div} u \rightarrow 1_{\{\rho=1\}} \operatorname{div} u \quad \text { a.e, }
$$

and since $\left|\rho^{k} \operatorname{div} u\right| \leq \operatorname{div} u \in L_{l o c}^{2}((0, T) \times \Omega)$, we get that div $u=0$ a.e. on $\{\rho=1\}$. This completes the proof of the lemma.

Next, we concentrate on the estimates we can derive from the equations. As is often the case when dealing with weak solutions, a priori bounds are obtained by making formal computations on the equation. Of course, those computations are rigorous if we assume that $(\rho, u, \pi)$ are smooth enough.

Since $0 \leq \rho \leq 1$ and $\int \rho(t)=\int \rho^{0}$, we see that $\rho \in L^{\infty}\left(0, T ; \mathrm{L}^{\infty} \cap\right.$ $L^{1}(\Omega)$ ). Then, the continuity of $\rho$ in $L^{p}(\Omega)-w$ is deduced from the bound on $\partial_{t} \rho$ in $L^{\infty}\left(H^{-1}\right)$, using the appendix $\mathrm{C}$ of [7]. The continuity of $\rho$ in $L^{p}(\Omega)$ (for $1 \leq p<\infty$ ) is then a simple consequence of the conservation of the mass and of the $L^{\infty}$ bound. Indeed, taking $\beta(\rho)=\sqrt{\beta}$ in (21), we get

$$
\frac{\partial \sqrt{\rho}}{\partial t}+\operatorname{div}(\sqrt{\rho} u)=\frac{1}{2} \sqrt{\rho} \operatorname{div} u
$$

In fact, we must approximate $\sqrt{\rho}$ by the following $C^{1}$ functions $\beta_{\varepsilon}=\sqrt{\rho+\varepsilon}$ and then observe that

$$
\beta_{\varepsilon}(\rho)-\rho \beta_{\varepsilon}^{\prime}(\rho)=\sqrt{\rho+\varepsilon}-\frac{\rho}{2 \sqrt{\rho+\varepsilon}} \rightarrow \frac{1}{2} \sqrt{\rho} \text { in } L^{2} .
$$

We deduce then that $\sqrt{\rho} \in C\left([0, T] ; L^{2}-w\right)$ and since

$$
\int_{\Omega}(\sqrt{\rho})^{2}(t)=\int \rho(t)=M
$$

is independent of $t$ we see that $\sqrt{\rho} \in C\left([0, T] ; L^{2}\right)$, hence $\rho \in C\left([0, T] ; L^{1}\right)$ and then we conclude using the $L^{\infty}$ bound on $\rho$.

Then, multiplying the momentum equation (2) by $u$ and using (1), we get (at least formally),

$$
\left\{\begin{array}{l}
\partial_{t}\left[\rho \frac{|u|^{2}}{2}\right]+\operatorname{div}\left(\rho u \frac{|u|^{2}}{2}+\pi u\right)-\pi \operatorname{div} u-\mu \Delta \frac{|u|^{2}}{2}+ \\
-\xi \operatorname{div}(u \operatorname{div} u)+\mu(D u)^{2}+\xi(\operatorname{div} u)^{2}=\rho f \cdot u
\end{array}\right.
$$

Vol. 16, n 3-1999. 
integrating over $\Omega$ and using the fact that $\pi$ div $u=0$ (since $\operatorname{div} u=$ 0 a.e. on $\{\rho=1\}$ and $\pi=0$ a.e. on $\{\rho<1\}$ ), we get the following energy identity

$$
\frac{\partial}{\partial t} \int_{\Omega} \rho \frac{|u|^{2}}{2}+\int_{\Omega} \mu(D u)^{2}+\xi(\operatorname{div} u)^{2}=\int_{\Omega} \rho f \cdot u
$$

and then integrating over $(0, t)$, we obtain

$$
\int_{\Omega} \rho \frac{|u|^{2}}{2}(t)+\int_{0}^{t} \int_{\Omega} \mu(D u)^{2}+\xi(\operatorname{div} u)^{2}=\int_{\Omega} \rho \frac{|u|^{2}}{2}(0)+\int_{0}^{t} \int_{\Omega} \rho f . u .
$$

Since $\mu+\xi>0$, we obtain (7) integrating by parts and using the Cauchy-Schwarz inequality,

$$
\begin{aligned}
\int_{\Omega}(\operatorname{div} u)^{2} & =\int_{\Omega} \partial_{i} u_{i} \partial_{j} u_{j}=\int_{\Omega} \partial_{j} u_{i} \partial_{i} u_{j} \\
& \leq \int_{\Omega} \sum_{i . j}\left(\partial_{i} u_{j}\right)^{2}=\int_{\Omega}(D u)^{2}
\end{aligned}
$$

Hence, there exists $\nu>0(=\inf (\mu, \mu+\xi))$ such that we have for almost all $t$ the following inequality where we have assumed in addition that $f \in L^{1}\left(\left(0, T ; L^{2}\right)\right.$,

$\int_{\Omega} \rho \frac{|u|^{2}}{2}(t)+\int_{0}^{t} \int_{\Omega} \nu(D u)^{2} \leq \int_{\Omega} \rho^{0} \frac{\left|u^{0}\right|^{2}}{2}+\int_{0}^{t}\|\sqrt{\rho}\|_{L^{\infty}}\|f\| L_{L^{2}}\|\sqrt{\rho} u\|_{L^{2}}$.

Using Gronwall's inequality and noticing that $\|\sqrt{\rho}\|_{L^{\infty}} \leq 1$, we deduce the first part of (8) and the bound on $D u$ in $L^{2}\left(0, T ; L^{2}(\Omega)\right)$. Then we get a bound on $\rho u$ in $L^{\infty}\left(0, T: L^{2}\right)$, sincc $\rho \in L^{\infty}$.

Next, we give the necessary changes to handle force terms satisfying (force). Let us set $f=f_{1}+f_{2}+f_{3}$ such that $f_{1} \in L^{1}\left(0, T ; L^{2}(\Omega)\right.$ ), $f_{2} \in L^{1}\left(0, T ; L^{\infty}(\Omega)\right)$ and $f_{3} \in L^{2}(0, T ; X)$, where $X=L^{\prime}(\Omega)$ if $\Omega=\mathbf{T}^{2}$, $X=L^{r}(B)$ if $\Omega=\mathbb{R}^{2}$ and $X=L^{\frac{2 N}{N+2}}(\Omega)$ if $N \geq 3$. Then, we see that $f_{1}$ can be treated as in the case when $f=f_{1} \in L^{1}\left(0, T ; L^{2}\right)$, the second term $f_{2}$ (which can be taken null in the periodic case) is treated as follows,

$$
\left|\int_{\Omega} \rho f_{2} \cdot u\right| \leq\|\rho\|_{L^{1}}^{1 / 2}\left\|f_{2}\right\|_{L^{\infty}}\|\sqrt{\rho} u\|_{L^{2}}
$$

And we use to conclude the conservation of the mass, namely $\|\rho\|_{L^{1}}=M$.

For the third term, we need to use the bound on $D u$ in $L^{2}$. 
If $\Omega=\mathrm{T}^{2}$, then we have

$$
\|u-f u\|_{L^{r^{\prime}}} \leq C\|D u\|_{L^{2}}
$$

where $\frac{1}{r}+\frac{1}{r^{\prime}}=1$ and hence

$$
\begin{aligned}
\left|\int_{\Omega} \rho f_{3} \cdot(u-f u)\right| & \leq C\|\rho\|_{L^{\infty}}\left\|f_{3}\right\|_{L^{r}}\|D u\|_{L^{2}} \\
& \leq \frac{C}{\varepsilon}\left\|f_{3}\right\|_{L^{r}}^{2}+\varepsilon\|D u\|_{L^{2}}^{2}
\end{aligned}
$$

On the other hand, we have

$$
M f u=\int \rho u-\int \rho(u-f u)
$$

and since $M>0$, we deduce that for almost all $t$

$$
|f u| \leq\|\sqrt{\rho} u\|_{L^{2}}+C\|\rho\|_{L^{2}}\|D u\|_{L^{2}}
$$

which yields

$$
\left|\int_{\Omega} \rho f_{3} \cdot(f u)\right| \leq C\left\|f_{3}\right\|_{L^{1}}\left(\|\sqrt{\rho} u\|_{L^{2}}+\|D u\|_{L^{2}}\right),
$$

and finally we deduce

$$
\left|\int_{\Omega} \rho f_{3} \cdot u\right| \leq \frac{C}{\varepsilon}\left\|f_{3}\right\|_{L^{\prime}}^{2}+\|\sqrt{\rho} u\|_{L^{2}}^{2}+\varepsilon\|D u\|_{L^{2}}^{2}
$$

Then, taking $\varepsilon$ small enough $(\varepsilon \leq \nu / 2)$, we can absorb the second term of the right hand side in $\nu\|D u\|_{L^{2}}^{2}$. Applying Gronwall's inequality, we conclude easily as before.

If $\Omega=\mathbb{R}^{2}$, then $f_{3}$ is assumed to be bounded in $L^{2}\left(0, T ; L^{r}(B)\right)$ for some fixed ball $B$, hence we have

$$
\int_{\Omega} \rho f_{3} \cdot u=\int_{B} \rho f_{3} \cdot u=\int_{B_{R}} \rho f_{3} \cdot\left(u-f_{B_{R}} u\right)+\int_{B_{R}} \rho f_{3} \cdot f_{B_{R}} u
$$

where $B_{R}$ is a big enough ball (the radius $R$ will be chosen later on). Next we know, using the classical Sobolev inequalities, that a bound on $D h$ in $L^{2}\left(B_{1}\right)$ yields a bound on $h-f h$ in $L^{2}\left(L^{q}\left(B_{1}\right)\right)$ for all $2 \leq q<+\infty$

$$
\left\|h-f_{B_{1}} h\right\|_{L^{q}\left(B_{1}\right)} \leq C_{q}\|D h\|_{L^{2}\left(B_{1}\right)}
$$

Vol. 16. $\mathrm{n}^{\circ}$ 3-1999. 
then by a scaling argument we get that

$$
\left\|h-f_{B_{R}} h\right\|_{L^{q}\left(B_{R}\right)} \leq C_{q} R^{2 / q}\|D h\|_{L^{2}\left(B_{R}\right)} .
$$

In fact, let $h$ be defined on $B_{1}$ and $h_{R}$ defined on $B_{R}$ by $h_{R}(x)=h\left(\frac{x}{R}\right)$, then we have

$$
\begin{aligned}
\int_{B_{R}}\left|h_{R}-f_{B_{R}} h_{R}\right|^{q} d x & =\int_{B_{1}}\left|h-f_{B_{1}} h\right|^{q} R^{2} d y \\
& \leq C_{q}^{q} R^{2} \|\left. D h\right|_{L^{2}\left(B_{1}\right)} ^{q} \\
\int_{B_{R}}\left|D\left(h_{R}\right)\right|^{2} d x & =\frac{1}{R^{2}} \int_{B_{R}}\left|D h\left(\frac{x}{R}\right)\right|^{2} d x \\
& =\int_{B_{1}}|D h(y)|^{2} d y
\end{aligned}
$$

We then get as in the periodic case,

$$
\begin{aligned}
\left|\int_{B_{R}} \rho f_{3} \cdot\left(u-f_{B_{R}} u\right)\right| & \leq C\|\rho\|_{L^{\infty}}\left\|f_{3}\right\|_{L^{r}}\left\|u-f_{B_{R}} u\right\|_{L^{r^{\prime}}} \\
& \leq C R^{2 / r^{\prime}}\|\rho\|_{L^{\infty}}\left\|f_{3}\right\|_{L^{r^{\prime}}}\|D u\|_{L^{2}} \\
& \leq \frac{C}{\varepsilon} R^{4 / r^{\prime}}\|\rho\|_{L^{\infty}}^{2}\left\|f_{3}\right\|_{L^{r}}^{2}+\varepsilon\|D u\|_{L^{2}}
\end{aligned}
$$

where $\frac{1}{r^{\prime}}+\frac{1}{r}=1$ (in the sequel we shall assume that $r^{\prime} \geq 4$, which means that $r \leq 4 / 3$, this assumption can be made without loss of generality since $B$ is bounded). In order to complete the estimates we need a bound on $f_{B_{R}} u$. For this we shall use the fact that the fluid does not flow very fast to infinity, indeed arguing as in [6] we can take a cut-off function $\phi \in C_{0}^{\infty}\left(\mathbb{R}^{\mathrm{N}}\right)$ such that $\phi=0$ if $|x| \geq 2, \phi=1$ if $|x| \leq 1,0 \leq \phi \leq 1$, then we multiply (1) by $\phi\left(\frac{x}{R}\right)$ and integrate by parts in $x$ to find

$$
\begin{aligned}
\frac{d}{d t} \int_{\mathbb{R}^{2}} \rho \phi\left(\frac{x}{R}\right) d x & =\int_{\mathbb{R}^{2}} \frac{1}{R} \rho u \cdot \nabla \phi\left(\frac{x}{R}\right) d x \\
& \geq-\frac{1}{R} C\|\nabla \phi\|_{L^{\infty}}\|\sqrt{\rho}\|_{L^{2}}\|\sqrt{\rho} u\|_{L^{2}}
\end{aligned}
$$

then taking $R$ big enough, we get for all $t$

$$
\int_{B_{R}} \rho d x \geq \frac{M}{2}-\frac{C M}{R} \int_{0}^{t}\|\sqrt{\rho} u\|_{L^{2}},
$$


hence, as long as

$$
\sup _{0 \leq t \leq T}\|\sqrt{\rho} u\|_{L^{2}} \leq \frac{R}{4 C T}
$$

we have

$$
\inf _{0 \leq t \leq T} \int_{B_{R}} \rho d x \geq \frac{M}{4}
$$

and we will show that this holds true if $R$ is chosen big enough. In the sequel, we assume that (34) holds, hence computing as in the periodic case, we get

$$
\begin{aligned}
\frac{M}{4} f_{B_{R}} u & \leq \int_{B_{R}} \rho u-\int_{B_{R}} \rho\left(u-f_{B_{R}} u\right) \\
& \leq\|\sqrt{\rho}\|_{L^{2}}\|\sqrt{\rho} u\|_{L^{2}}+\|\rho\|_{L^{q}}\left\|u-f_{B_{R}} u\right\|_{L^{q^{\prime}}} \\
& \leq\|\sqrt{\rho}\|_{L^{2}}\|\sqrt{\rho} u\|_{L^{2}}+\|\rho\|_{L^{q}} R^{2 / q^{\prime}}\|D u\|_{L^{2}} \\
& \leq\|\sqrt{\rho}\|_{L^{2}}\|\sqrt{\rho} u\|_{L^{2}}+\frac{C}{\varepsilon}\|\rho\|_{L^{q}}^{2} R^{4 / q^{\prime}}+\varepsilon\|D u\|_{L^{2}}^{2}
\end{aligned}
$$

where $\frac{1}{q^{\prime}}+\frac{1}{q}=1, q^{\prime}>4$ (for instance we can take $q^{\prime}=r^{\prime}$ ). Hence, the second term can be estimated as follows

$$
\begin{aligned}
\left|\int_{B_{R}} \rho f_{3} \cdot f_{B_{R}} u\right| \leq\|\rho\|_{L^{\infty}} \frac{4}{M}\left[\|\sqrt{\rho}\|_{L^{2}}\|\sqrt{\rho} u\|_{L^{2}}\right. \\
\left.+\|\rho\|_{L^{r}} R^{2 / r^{\prime}}\|D u\|_{L^{2}}\right]\left\|f_{3}\right\|_{L^{1}} \\
\leq C\|\sqrt{ } \rho u\|_{L^{2}}^{2}+C R^{4 / r^{\prime}}\left\|f_{3}\right\|_{L^{r}(B)}^{2}+\varepsilon\|D u\|_{L^{2}}^{2} .
\end{aligned}
$$

Summing up the two estimates, we get

$$
\left|\int_{\Omega} \rho f_{3} \cdot u\right| \leq \frac{C}{\varepsilon} R^{4 / r^{\prime}}\left\|f_{3}\right\|_{L^{r}}^{2}+\|\sqrt{\rho} u\|_{L^{2}}^{2}+\varepsilon\|D u\|_{L^{2}}^{2}
$$

where $C$ is a constant that does not depend on $R$ and where (35) is true as long as (34) holds. Taking $\varepsilon$ small enough $(\varepsilon \leq \nu / 2)$, we can absorb the second term of the right hand side in $\nu\|D u\|_{L^{2}}^{2}$. Then, taking $R$ large enough we conclude easily.

If $N \geq 3$ then we have

$$
\|u\|_{L^{\frac{2 N}{N-2}}} \leq C\|D u\|_{L^{2}}
$$


and hence

$$
\left|\int_{\Omega} \rho f_{3} \cdot u\right| \leq C\left\|f_{3}\right\|_{L^{\frac{2 N}{N+2}}}\|u\|_{L^{\frac{2 N}{N-2}}} \leq \frac{C}{\varepsilon}\left\|f_{3}\right\|_{L^{\frac{2 N}{N+2}}}^{2}+\varepsilon\|D u\|_{L^{2}}^{2},
$$

and we conclude easily.

Next, in order to deduce the $L^{1}$ bound on $\pi$ (we assume that $\pi \in L^{1}$ since $\pi$ will be the weak limit of a bounded sequence in $L^{1}$ ), we apply the operator $(-\Delta)^{-1} \operatorname{div}$ to the momentum equation (this idea was used by P.-L. Lions in the case of compressible Navier-Stokes equations). We begin by the case $\Omega=\mathbb{R}^{\mathrm{N}}$,

$$
\left\{\begin{array}{c}
\pi=\partial_{t}(-\Delta)^{-1} \operatorname{div}(\rho u)-R_{i} R_{j}\left(\rho u_{i} u_{j}\right)+ \\
+(\mu+\xi) \operatorname{div}(u)+(-\Delta)^{-1} \operatorname{div}(\rho f)
\end{array}\right.
$$

where $R_{i}$ is the Riesz transform $\left(R_{i}=\Delta^{-1} \partial_{i}\right)$ and where we must fulfill the summation over $i$ and $j$. Here wc have used that $\Delta^{-1}[\Delta(\operatorname{div} u)]=\operatorname{div} u$ and that $\Delta^{-1}[\Delta \pi]=\pi$, since we assume that $\operatorname{div} u \in L^{2}\left(\mathbb{R}^{N}\right)$ a.e. on $t$ and that $\pi \in L^{1}\left(\mathbb{R}^{N}\right)$ a.e. on $t$. In order to obtain bounds which depend only on the initial data, we can integrate this identity and use the fact that $\pi \geq 0$, but since we have only an $L^{2}$ bound on div $u$, we cannot integrate on the whole space. To recover this, we use the fact that $\rho \pi=\pi$ and multiply (37) by $\rho$,

$$
\left\{\begin{aligned}
\pi & =\partial_{t}\left[\rho(-\Delta)^{-1} \operatorname{div}(\rho u)\right]+\operatorname{div}\left[\rho u(-\Delta)^{-1} \operatorname{div}(\rho u)\right]+ \\
& +\rho u_{i} R_{i} R_{j}\left(\rho u_{j}\right)-\rho R_{i} R_{j}\left(\rho u_{i} u_{j}\right)+ \\
& +(\mu+\xi) \rho \operatorname{div}(u)+\rho(-\Delta)^{-1} \operatorname{div}(\rho f) .
\end{aligned}\right.
$$

Integrating (38) over $\mathbb{R}^{\mathrm{N}}$, we get a bound in $L^{1}$ that depends only on the initial data. We are going to explain how we treat the six terms that occur in the right hand side of (38). First we have

$$
\left|\int_{0}^{T} \int_{\mathbb{R}^{\mathrm{N}}} \partial_{t}\left[\rho(-\Delta)^{-1} \operatorname{div}(\rho u)\right]\right| \leq 2\left\|\rho(-\Delta)^{-1} \operatorname{div}(\rho u)\right\|_{L^{\infty}\left(0, T ; L^{1}(\Omega)\right)},
$$

then using that $\rho u \in L^{\infty}\left(L^{3 / 2}\right)$, we get $(-\Delta)^{-1} \operatorname{div}(\rho u) \in L^{\infty}\left(L^{q}\right)$, with $\frac{1}{q}=\frac{2}{3}-\frac{1}{N}>0$, and then we conclude by using that $\rho \in L^{\infty}\left(L^{q^{\prime}}\right)$, where $\frac{1}{q}+\frac{1}{q^{\prime}}=1$, 


$$
\left|\int_{0}^{T} \int_{\mathbb{R}^{\mathrm{N}}} \partial_{t}\left[\rho(-\Delta)^{-1} \operatorname{div}(\rho u)\right]\right| \leq 2\|\rho\|_{L^{\infty}\left(L^{q^{\prime}}\right)}\|\rho u\|_{L^{\infty}\left(L^{3 / 2}\right)}
$$

Notice that we cannot use the continuity of $\rho u$ in $L^{p}-w(1 \leq p \leq 2)$ at this stage (since $\pi \in \mathcal{M}$ ) and that we must explain the meaning of (39). Let $\phi_{n}$ be a sequence of $C_{c}^{\infty}(0, T)$ that converges to $1_{[0, T]}$ in $L^{1}(\mathbb{R})$ (and in $L^{q}(\mathbb{R})$ for all $\left.1 \leq q \leq \infty\right)$ and such that $\phi_{n}$ is nondecreasing on $\left[0, \frac{1}{n}\right]$, increasing on $\left[T-\frac{1}{n}, T\right]$ and $\phi_{n}(t)=1$ on $\left[\frac{1}{n}, T-\frac{1}{n}\right]$. Then we get

$$
\begin{aligned}
\left|\int_{0}^{T} \int_{\mathbb{R}^{\mathrm{N}}} \partial_{t}\left[\rho(-\Delta)^{-1} \operatorname{div}(\rho u)\right] \phi_{n}(t)\right| & =\left|\int_{0}^{T} \int_{\mathbb{R}^{\mathrm{N}}}\left[\rho(-\Delta)^{-1} \operatorname{div}(\rho u)\right] \partial_{t} \phi_{n}(t)\right| \\
& \leq C\left\|\partial_{t} \phi_{n}(t)\right\|_{L^{1}(0, T)} \\
& \leq C\left[\int_{0}^{1 / n} \partial_{t} \phi_{n}(t)-\int_{T-1 / n}^{T} \partial_{t} \phi_{n}(t)\right] \\
& =2\left\|\rho(-\Delta)^{-1} \operatorname{div}(\rho u)\right\|_{L^{\infty}\left(0, T ; L^{1}(\Omega)\right)} .
\end{aligned}
$$

where $C=\left\|\rho(-\Delta)^{-1} \operatorname{div}(\rho u)\right\|_{L^{\infty}\left(0, T ; L^{1}(\Omega)\right)}$.

Next, we explain why the integration over $\mathbb{R}^{N}$ of the second term vanishes by using a cut-off function, let $\phi \in C_{c}^{\infty}\left(\mathbb{R}^{N}\right), 0 \leq \phi \leq 1, \phi=1$ on $B_{1}, \phi=0$ on $\mathbb{R}^{\mathrm{N}}-\mathrm{B}_{2}$, then for any $R \in(1,+\infty)$, we have, setting $U=\rho u(-\Delta)^{-1} \operatorname{div}(\rho u)$,

$$
\begin{aligned}
\left|\int_{\mathbb{R}^{N}} \operatorname{div} U \phi\left(\frac{x}{R}\right) d x\right| & =\left|\int_{\mathbb{R}^{N}} U \cdot \frac{1}{R} \nabla \phi\left(\frac{x}{R}\right) d x\right| \\
& \leq\|U\|_{L^{1}\left(\mathbb{R}^{N}\right)} \frac{1}{R}\|\nabla \phi\|_{L^{\infty}\left(\mathbb{R}^{N}\right)}
\end{aligned}
$$

and we conclude by letting $R$ tend to $+\infty$. We only need to show that $U \in L^{\infty}\left(0, T ; L^{1}\right)$, which can be deduced from the bound we have on $\rho u$ in $L^{\infty}\left(0, T ; L^{q}\right)$, where $\frac{1}{q}+\left(\frac{1}{q}-\frac{1}{N}\right)=1(1<q \leq 2)$.

The third and the fourth terms are handled similarly if $N \geq 3$. In fact, since $\rho|u|^{2} \in L^{1}\left(0, T ; L^{s}\right) \quad\left(s=\frac{N}{N-2}>1\right)$ and since the Riesz transforms are bounded in $L^{p}$ for $1<p<+\infty$, we see that $R_{i} R_{j}\left(\rho u_{i} u_{j}\right) \in L^{1}\left(0, T ; L^{s}\right)$ and then using the fact that $\rho \in L^{\infty}\left(0, T ; L^{s^{\prime}}\right)$, we see that $\rho R_{i} R_{j}\left(\rho u_{i} u_{j}\right) \in L^{1}$. The third term is treated in the same way Vol. $16, \mathrm{n}^{\circ} 3-1999$. 
and is in fact simpler. However if $N=2$, the fourth term cannot be treated by this method since we no longer know that $\rho|u|^{2} \in L^{1}\left(0, T ; L^{s}\right)$ for some $s>1$. Nevertheless, since $D u \in L^{2}\left(0, T ; \mathbb{R}^{2}\right)$, we see (as in P.-L. Lions [6]) that $u \in L^{2}(0, T ; B M O)$. In fact for any cube $Q$ in $\mathbb{R}^{2}$, we have

$$
\begin{aligned}
\int_{Q}\left|u-f_{Q} u\right| d x & \leq|Q|^{1 / 2}\left(\int_{Q}\left|u-f_{Q} u\right|^{2} d x\right)^{1 / 2} \\
& \leq C|Q|\left(\int_{Q}|D u|^{2} d x\right)^{1 / 2} \\
& \leq C|Q| \int_{\mathbb{R}^{2}}|D u|^{2} d x
\end{aligned}
$$

Hence using the Coifman-Rochberg-Weiss commutator theorem [3], we get that $\left[u, R_{i} R_{j}\right]$ is bounded in $L^{p}$ for $1<p<+\infty$, so we have the following estimate

$$
\begin{gathered}
\left\|R_{i} R_{j}\left(\rho u_{i} u_{j}\right)-u_{i} R_{i} R_{j}\left(\rho u_{j}\right)\right\|_{L^{2}\left(0, T ; L^{2}\right)} \\
\leq C\|u\|_{L^{2}(0, T ; B M O)}\|\rho u\|_{L^{\infty}\left(0, T ; L^{2}\right)} .
\end{gathered}
$$

Next, we use that $\rho \in L^{2}\left(0, T ; L^{2}\right)$ to deduce the desired $L^{1}$ bound.

For the fifth, we have the following straightforward computation

$$
\mid \int_{0}^{T} \int_{\mathbb{R}^{N}} \rho \text { div } u \mid \leq\|\rho\|_{L^{2}\left(0, T ; L^{2}\right)} \| \text { div } u \|_{L^{2}\left(0, T ; L^{2}\right)} .
$$

Finally, for the sixth term we have merely

$$
\left|\int_{0}^{T} \int_{\mathbb{R}^{\mathrm{N}}}\right| \rho(-\Delta)^{-1} \operatorname{div}(\rho f) \mid \leq\|\rho\|_{L^{\infty}\left(L^{q^{\prime}}\right)}\|\rho f\|_{L^{1}\left(L^{r}\right)}
$$

where $\frac{1}{q^{\prime}}+\frac{1}{r}-\frac{1}{N}=1$ since of $\in L^{1}\left(L^{r}\right)$ and $\left(\|\rho f\|_{L^{\prime}\left(L^{r}\right)} \leq\right.$ $C\|f\|_{L^{1}\left(L^{2}\right)+L^{2}\left(L^{r}\right)}$ with $\left.r<2\right)$.

Next, we explain the changes in the above argument we must perform in the periodic case. Now, (37) is replaced by

$$
\left\{\begin{aligned}
\pi-f \pi= & \partial_{t}(-\Delta)^{-1} \operatorname{div}(\rho u)-R_{i} R_{j}\left(\rho u_{i} u_{j}\right)+ \\
& +(\mu+\xi) \operatorname{div}(u)+(-\Delta)^{-1} \operatorname{div}(\rho f)
\end{aligned}\right.
$$

Before integrating over $\mathbf{T}^{\mathrm{N}}$, we multiply (as in the case of $\mathbb{R}^{\mathrm{N}}$ ) by $\rho$. Notice however that we do so for different reasons than in the whole space case. 
In fact, since $\mathbf{T}^{\mathrm{N}}$ is a bounded domain $\operatorname{div}(u) \in L^{1}((0, T) \times \Omega)$ and we can integrate (37) without any problem, but this integration gives no estimates on $\pi$, since the integral of $\pi-f \pi$ vanishes. This is why we multiply by $\rho$,

$$
\left\{\begin{aligned}
\pi-\rho f \pi & =\partial_{t}\left[\rho(-\Delta)^{-1} \operatorname{div}(\rho u)\right]+\operatorname{div}\left[\rho u(-\Delta)^{-1} \operatorname{div}(\rho u)\right]+ \\
& +\rho u_{i} R_{i} R_{j}\left(\rho u_{j}\right)-\rho R_{i} R_{j}\left(\rho u_{i} u_{j}\right)+ \\
& +\rho(\mu+\xi) \operatorname{div}(u)+\rho(-\Delta)^{-1} \operatorname{div}(\rho f)
\end{aligned}\right.
$$

And integrating (43) over $\mathbf{T}^{\mathrm{N}}$, we get

$$
(1-M) \int_{0}^{T} \int_{\mathrm{T}^{\mathrm{N}}} \pi \leq C\left(\rho^{0}, m^{0}\right)
$$

in fact the estimates here are simpler than in the whole space case, and since $M<1$, we get the desired bound.

Now, we are going to prove theorem 1.1. From the bounds on $\rho, \rho u, u$, $\pi$ and in the case $N \geq 3$ or $\Omega=\mathrm{T}^{2}$ we deduce that,

$$
\left\{\begin{array}{l}
\rho \in C\left([0, T] ; L^{p}\right) \cap C^{1}\left([0, T] ; H^{-1}\right), \\
\pi \in W^{-1, \infty}\left(H^{1}\right)+L^{1}\left(L^{N /(N-2}\right) \cap L^{\alpha}\left(L^{\beta}\right)+L^{2}\left(L^{2}\right)+L^{1}\left(L^{q}\right),
\end{array}\right.
$$

where we have used (37) to deduce the bounds on $\pi$ and where $1<\alpha, \beta<\infty$ and $\frac{1}{\beta}=\frac{1}{\alpha} r+\left(1-\frac{1}{\alpha}\right), r=\frac{N \cdot 2}{N}$ if $N \geq 3$ and $1<r<\infty$ if $N=2, \frac{1}{q}=\frac{1}{r}-\frac{1}{N}$.

Then, $\pi$ can be rewritten as $\pi=\partial_{t} h+\pi_{2}+\pi_{3}+\pi_{4}$ where $h \in L^{\infty}\left(H^{1}\right)$. The terms $\rho \pi_{2}, \rho \pi_{3}, \rho \pi_{4}$ are well-defined. To give a meaning to $\rho \partial_{t} h$, we use that $\rho=d i v_{x} g$ where $g \in C^{1}\left(0, T ; L^{2}\right)^{N}$. Hence, we can write (and define $\rho \pi_{1}$ as follows)

$$
\begin{aligned}
\rho \pi_{1} & =d i v_{x} g \partial_{t} h \\
& =d i v_{x} \partial_{t}[g h]-d i v_{x}\left[h \partial_{t} g\right]-\partial_{t}\left[g d i v_{x} h\right]+\partial_{t} d i v_{x} h
\end{aligned}
$$

Next, in order to show that $\pi \in \mathcal{M}\left(0, T ; L^{1}(\Omega)\right)$, we only need to prove that

$$
\lim _{|\omega| \rightarrow 0} \int_{0}^{T} \int_{\omega} \pi=0
$$

which can be deduced easily from (45). 
Since $\pi \in \mathcal{M}$, it is easy to see that $\rho u \in B V\left(0, T ; H^{-m}\right)$ for $m$ large enough. Then, since $\rho u \in L^{\infty}\left(0, T: L^{2}\right)$, we deduce that $p u \in B V\left(0, T ; L^{2}-w\right)$. We recall here that the values taken by $\rho u$ on $[0, T]$ belong to a fixed ball $B_{R}$ of $L^{2}(\Omega)$ and that we can equip $B_{R}$ with a "weak topology" distance $d$. Then $\phi \in B V\left([0, T] ;\left(B_{R}, d\right)\right)$ if and only if the following supremum is finite

$$
\|\phi\|_{B V\left(0, T: L^{2}-w\right)}=\sup _{\substack{n=1 \in \mathbb{N} \\ 0=t_{0}<t_{1}<t_{n} \leq T}} \sum_{i=0}^{n} d\left(\phi\left(t_{i+1}, \phi\left(t_{i}\right)\right) .\right.
$$

Finally to deduce the continuity of $P(\rho u)$, we apply the operator $P$ to the momentum equation and get

$$
\frac{P(\partial \rho u)}{\partial t}+P(\operatorname{div}(\rho u \otimes u))-\mu \Delta P(u)=P(\rho f) .
$$

Then, the continuity in the weak topology is deduced from the following bound on $\frac{\partial P(\rho u)}{\partial t}$ and the Appendix $\mathrm{C}$ of [7]. In deed, we have $\frac{\partial P(\rho u)}{\partial t} \in L^{\infty}\left(0, T ; W^{-1,1}\right)+L^{2}\left(0, T ; H^{-1}\right)+L^{1}\left(0 . T ; L^{2}\right)+L^{2}\left(0, T ; L^{r}\right)$.

\section{COMPACTNESS}

In this section, we are concerned with a sequence of weak solutions $\left(\rho_{n}, u_{n}, \pi_{n}\right)$ of (1)-(4), with $f=0$ for simplicity. This sequence is assumed to exist even though we have not proved yet existence results for (1) - (4). The sequence $\left(\rho_{n}, u_{n}, \pi_{n}\right)$ satisfies, uniformly in $n$, the a priori estimates derived in the previous section and the following initial conditions

$$
\left.\rho_{n} u_{n}\right|_{t=0}=m_{n}^{0},\left.\rho_{n}\right|_{t=0}=\rho_{n}^{0}
$$

where $0 \leq \rho_{n}^{0} \leq 1$ a.e., $\rho_{n}^{0}$ is bounded in $L^{1}(\Omega), m_{n}^{0}$ is bounded in $L^{2}(\Omega)$, $m_{n}^{0}=0$ a.e. on $\left\{\rho_{n}^{0}=0\right\}, \rho \not \equiv 0$ and $\rho_{n}^{0}\left|u_{n}^{0}\right|^{2}$ is bounded in $L^{1}$, denoting by $u_{n}^{0}=\frac{m_{n}^{0}}{\rho_{n}^{0}}$ on $\left\{\rho_{n}^{0}>0\right\}, u_{n}^{0}=0$ on $\left\{\rho_{n}^{0}=0\right\}$. In the case of $\mathrm{T}^{\mathrm{N}}$, we also assume that $f \rho_{n}^{0}=M$, for some fixed $M$ (or some $M_{n}, 0<M_{n}<1$ such that $M_{n} \rightarrow M$ ), with $0<M<1$.

Without loss of generality, extracting subsequences if necessary also denoted by $\left(\rho_{n}, u_{n}, \pi_{n}\right)$, we can assume that $\left(\rho_{n}, u_{n}, \pi_{n}\right)$ converge to 
some $(\rho, u, \pi)$ in the sense of distributions. More precisely, we can assume that $\rho_{n} \rightarrow \rho$ weakly in $L^{p}((0, T) \times \Omega)$ for any $1<p<+\infty$, $\rho \in L^{\infty}\left(0, T, L^{1} \cap L^{\infty}(\Omega)\right)$ and $0 \leq \rho \leq 1, u_{n} \rightarrow u$ weakly in $L^{2}\left(0, T: H^{1}(B)\right)$, where $B=\mathbf{T}^{\mathrm{N}}$ if $\Omega=\mathbf{T}^{\mathrm{N}}$ and $B$ is any ball in $\mathbb{R}^{\mathrm{N}}$ if $\Omega=\mathbb{R}^{\aleph}, D u_{n} \rightarrow D u$ weakly in $L^{2}\left(0, T ; L^{2}(\Omega)\right)$. Finally, we may assume that $\pi_{n} \rightarrow \pi$ weakly in $\mathcal{M}((0, T) \times \Omega)$, where $\pi$ is a positive measure.

THEOREM 3.1. - Under the above assumptions, we have

$$
\begin{gathered}
\rho_{n} u_{n} \rightarrow \rho u \quad \text { weakly star in } L^{\infty}\left(0, T^{\prime} ; L^{2}(\Omega)\right) \\
\rho_{n} u_{n} \otimes u_{n} \rightarrow \rho u \otimes u \quad \text { weakly in } L^{\alpha}\left(0, T ; L^{\beta}(\Omega)\right),
\end{gathered}
$$

where $1<\alpha, \beta<\infty$ and $\frac{1}{\beta}=\frac{1}{\alpha} r+\left(1-\frac{1}{\alpha}\right), r=\frac{N-2}{N}$ if $N \geq 3$ and $1<r<\infty$ if $N=2$, (if $\Omega=\mathbb{R}^{2}$, the convergence holds locally in space)

Remark. - In general $(\rho, u, \pi)$ is not a solution of (1)-(4), in fact condition (4) does not hold in all times. The homogeneisation example given in [6] for the case of the compressible Navier-Stokes equations can be adapted to our system. In the following theorem, we give a sufficient condition on the initial data, for $(\rho, u, \pi)$ to be a solution.

THEOREM 3.2. - If we assume in addition that $\rho_{n}^{0}$ converges to $\rho^{0}$ in $L^{1}(\Omega)$, then $(\rho, u, \pi)$ is a solution of $(1)-(4)$ and in addition the following strong convergences hold

$$
\begin{gathered}
\rho_{n} \rightarrow \rho \quad \text { in } \quad C\left(0, T ; L^{p}(\Omega)\right) \text { for any } 1 \leq p<+\infty, \\
\rho_{n} u_{n} \rightarrow \rho u \quad \text { in } \quad L^{p}\left(0, T ; L^{r}(\Omega)\right) \text { for any } 1 \leq p<+\infty, 1 \leq r<2, \\
\rho_{n} u_{n} \otimes u_{n} \rightarrow \rho u \otimes u \quad \text { in } \quad L^{p}\left(0, T ; L^{1}(\Omega)\right) \text { for any } 1 \leq p<+\infty
\end{gathered}
$$

this last convergence holds locally if $\Omega=\mathbb{R}^{2}$.

Remark. - Using the bound on $D u_{n}$ in $L^{2}$, we see that the strong convergence of $\rho_{n} u_{n}$ also holds in $L^{p}\left(0, T ; L^{r}(\Omega)\right)$ for any $p>2$ and $1 \leq r<\frac{2 p N}{p N-4}$ if $\Omega \neq \mathbb{R}^{2}$. If $\Omega=\mathbb{R}^{2}$ then this convergence is local in space.

For $\rho_{n} u_{n} \otimes u_{n}$ the convergence also holds in $L^{p}\left(0, T ; L^{q}(\Omega)\right.$ ) (locally if $\Omega=\mathbb{R}^{2}$ ) for any $p \geq 2$ and $1<q<\frac{p N}{p N-2}$.

Proof. - The proof of the two theorems is very close to the proof given in [6]. In fact theorem 3.1 is deduced from the following compensatedcompactness lemma, 
LEMMA 3.3. - Let $g^{n}, h^{n}$ converge weakly to $g, h$ respectively in $L^{p_{1}}\left(0, t ; L^{p_{2}}\right), L^{q_{1}}\left(0, t ; L^{q_{2}}\right)$, where $1 \leq p_{1}, p_{2} \leq+\infty, \frac{1}{p_{1}}+\frac{1}{q_{1}}=$ $\frac{1}{p_{2}}+\frac{1}{q_{2}}=1$. We assume in addition that

$$
\begin{aligned}
& \left\{\begin{array}{l}
\frac{\partial g^{n}}{\partial t} \quad \text { is bounded in } \quad \mathcal{M}\left(0, T ; W^{-m, 1}(\Omega)\right) \\
\text { for some } m \geq 0 \text { independent of } n
\end{array}\right. \\
& \left\|h^{n}\right\|_{L^{1}\left(0, T ; H^{s}\right)} \quad \text { is bounded for some } s>0 .
\end{aligned}
$$

Then, $g^{n} h^{n}$ converges to $g h$ in $\mathcal{D}^{\prime}$.

Next, let us observe that $\rho_{n}$ is bounded in $L^{\infty}((0, T) \times \Omega)$ and converges weakly to $\rho$ in $L^{p_{1}}\left(0, T ; L^{p_{2}}(\Omega)\right)$ for any $1 \leq p_{1}, p_{2}<+\infty$ and that $u_{n}$ converges weakly to $u$ in $L^{2}\left(0, T ; L^{\beta}\right)$ where $\beta=\frac{N}{N-2}$ if $N \geq 3$ and $2 \leq \beta<+\infty$ if $N=2$ (this holds locally in space if $\Omega=\mathbb{R}^{2}$ ). We can take $p_{1}=2$ and $p_{2}$ such that $\frac{1}{p_{2}}+\frac{1}{\beta}=1$. In order to apply the lemma 3.3 $\left(g^{n}=\rho_{n}, h^{n}=u_{n}\right)$, we need some compactness in time and in space which are straightforward since we know that $\frac{\partial \rho_{n}}{\partial t}$ is bounded in $L^{\infty}\left(0, T ; H^{-1}\right)$ and that $u_{n}$ is bounded in $L^{2}\left(0, T: H^{1}\right)$ (locally if $\left.\Omega=\mathbb{R}^{\mathrm{N}}\right)$. Hence, using the preceding lemma we obtain the weak convergence of $\rho_{n} u_{n}$ to $\rho u$ (in the sense of distributions).

Then, we apply the lemma to the following couple $\left(g^{n}=\rho_{n} u_{n}, h^{n}=u_{n}\right)$. In order to do so, we observe that $\rho_{n} u_{n}$ converges weakly to $\rho u$ in $I^{p_{1}}\left(0, T ; I^{p_{2}} \Omega\right)$ for any $1<p_{1}<+\infty, 1<p_{2} \leq 2$ (in fact convergence in the sense of distribution is equivalent to weak convergence in these spaces). We can take $p_{1}=2$ and $p_{2}$ such that $\frac{1}{p_{2}}+\frac{1}{\beta}=1$. Next we have in view of (2) the following bound

$\frac{\partial \rho_{n} u_{n}}{\partial t}$ bounded in $L^{\infty}\left(0, T ; W^{-1,1}\right)+L^{2}\left(0, T ; H^{-1}\right)+\mathcal{M}\left(0, T ; W^{-1-\varepsilon, 1}\right)$.

for any $\varepsilon>0$. Hence, applying lemma 3.3 once more, we deduce the weak convergence of $\rho_{n} u_{n} \otimes u_{n}$ to $\rho u \otimes u$ in the sense of distributions and hence in the spaces $L^{\alpha}\left(0, T ; L^{\beta}(\Omega)\right)$.

In conclusion, these weak convergences show that the limit $(\rho, u, \pi)$ satisfy the equation (1), (2), (3). In fact, it is obvious for the first 
and the second equation, for the third one we apply lemma 2.1 since $u \in L^{2}\left(0, T ; H_{l o c}^{1}\right)$ and that $0 \leq \rho \leq 1$. However in general (4) does not hold and we will see below that if the $\rho_{n}^{0}$ converges to $\rho^{0}$ then (4) holds and $(\rho, u, \pi)$ is a solution of the initial system.

Now, we turn to the proof of theorem 3.2. The idea of the proof relies on the use of some compactifying commutators. This idea was used by P.-L. Lions in [6]. In what follows we will give a sketch of the proof and omit the problems related to the justification of the computation. We refer to [6] for the missing justifications.

Taking $\beta(\rho)=\rho \log \rho$ in (21), we get

$$
\frac{\partial \rho \log (\rho)}{\partial t}+\operatorname{div}(\rho \log (\rho) u)=-\rho \operatorname{div} u .
$$

This equality follows from approximating $\rho \log (\rho)$ by the following $C^{1}$ functions $\beta_{\varepsilon}=\rho \log (\rho+\varepsilon)$ and observing that

$$
\beta_{\varepsilon}(\rho)-\rho \beta_{\varepsilon}^{\prime}(\rho)=-\rho^{2}(\rho+\varepsilon)^{-1} \rightarrow \rho \text { in } L^{2} .
$$

Next, we observe that this equality also holds for $\rho_{n}$ and that extracting subsequences if necessary we can assume that $\rho_{n} \log \rho_{n}$ converges weakly to $\bar{s}$ in $L^{p}((0, T) \times \Omega)$ for $1<p<+\infty$. In fact, since $0 \leq \rho_{n} \leq 1$, we see that for any $p>1$, there exists a constant $C_{p}$ (independent of $\mathrm{n}$ ) such that for all $n$, we have $\left|\rho_{n} \log \rho_{n t}\right|^{p} \leq C_{p} \rho_{n}$. Then, passing to the limit in the equation satisfied by $\rho_{n} \log \rho_{n}$, we obtain

$$
\frac{\partial \bar{s}}{\partial t}+\operatorname{div}(\bar{s} u)=-\overline{\rho \operatorname{div} u}
$$

where $\overline{\rho \operatorname{div} u}$ denotes the weak limit of $\rho_{n} \operatorname{div} u_{n}$. In fact we can apply lemma 3.3 to the pair $\left(g^{n}=\rho_{n} \log \rho_{n}, h^{n}=u_{n}\right)$, which yields the weak convergence of $\rho_{n} \log \rho_{n} u_{n}$ to $\bar{s} u$. We see then that $-\rho_{n} \operatorname{div} u_{n}$ converges weakly to the first hand side of (50). Let $s$ denote $\rho \log (\rho)$, then we get

$$
\frac{\partial}{\partial t}(\bar{s}-s)+\operatorname{div}[(\bar{s}-s) u]=-\overline{\rho \operatorname{div} u}+\rho \operatorname{div} u .
$$

Next, using the momentum equation we shall show that the second hand side of $(51)$ is actually equal to $\rho \pi-\pi$. Hence, integrating in $x$, we deduce

$$
\frac{\partial}{\partial t} \int_{\Omega}(\bar{s}-s) d x \leq 0
$$

Vol. 16, n 3- 1999. 
Let us then notice that, by standard convexity considerations, we obtain that $\bar{s} \geq s$ a.e., and since $\bar{s}-\left.s\right|_{t=0}=0$ we conclude that $\bar{s}=s$ for almost all $t$ in $(0, T)$. We also deduce at the same time that $\rho \pi=\pi$.

Now, we should only compute the right hand side of (51). We begin by the case of the whole space $\mathbb{R}^{\mathrm{N}}$, with $N \geq 3$ and then explain the changes that must be done in the other cases. Taking the divergence of (2), applying $(-\Delta)^{-1}$ and multiplying by $\rho$, we get the equation (38) written for $\left(\rho_{n}, u_{n}, \pi_{n}\right)$. Next passing to the limit and taking subsequences if necessary we get

$$
\left\{\begin{aligned}
\pi-(\mu+\xi) \overline{\rho \operatorname{din}(u)} & =\partial_{t} \overline{\left[\rho(-\Delta)^{-1} \operatorname{div}(\rho u)\right]} \\
& +\overline{\operatorname{div}\left[\rho u(-\Delta)^{-1} \operatorname{div}(\rho u)\right]} \\
& +\rho u_{i} R_{i} R_{j}\left(\rho u_{j}\right)-\rho R_{i} R_{j}\left(\rho u_{i} u_{j}\right)
\end{aligned}\right.
$$

where the $\bar{A}$ denotes the weak limit of $A_{n}$. Next, we apply the same computations for $(\rho, u, \pi)$ and we deduce

$$
\left\{\begin{aligned}
\rho \pi-(\mu+\xi) \rho \operatorname{din}(u) & =\partial_{t}\left[\rho(-\Delta)^{-1} \operatorname{div}(\rho u)\right] \\
& +\operatorname{cliv}\left[\rho u(-\Delta)^{-1} \operatorname{div}(\rho u)\right] \\
& +\rho u_{i} R_{i} R_{j}\left(\rho u_{j}\right)-\rho R_{i} R_{j}\left(\rho u_{i} u_{j}\right)
\end{aligned}\right.
$$

Let us notice that we cannot use the equation $/ \pi=\pi$. Next arguing as in [6], we show that the second terms in the right hand side of (52) and (53) coincide. In fact, using lemma 3.3, we see that $\rho_{n}(-\Delta)^{-1} \operatorname{div}\left(\rho_{n} u_{n}\right)$ weakly converges to $\rho(-\Delta)^{-1} \operatorname{div}(\rho u)$ and that $\rho_{n} u_{n}(-\Delta)^{-1} \operatorname{div}\left(\rho_{n} u_{n}\right)$ weakly converges to $\rho u(-\Delta)^{-1} \operatorname{div}(\rho u)$. The last term can be written as $\rho\left[u_{i}, R_{i} R_{j}\right] \rho u_{j}$, (where $\left[u_{i}, R_{i} R_{j}\right]$ denotes the commutator of $u_{i}$ and $R_{i} R_{j}$ ). Using the general results on commutator of this type of Bajanski and R. Coifman [1] and R. Coifman and Y. Meyer [2], we deduce that $\left[u_{n, i}, R_{i} R_{j}\right] p u_{n, j}$ is bounded in $L^{1}\left(0, T ; W^{1 . q}\right)$, where $\frac{1}{q}=\frac{1}{2}+\frac{1}{\beta}<1$, since $u \in L^{2}\left(0, T: H^{1}\right)$ (locally if $\Omega=\mathbb{R}{ }^{N}$ ) and $m u \in L^{2}\left(0, T ; L^{3}\right)$, where $\beta=\frac{2 N}{N-2}>2$ if $N \geq 3$ and $2<\beta<+\infty$ if $N=2$ (locally if $\Omega=\mathbb{R}^{2}$ ). Lemma 3.3 applies to this case too and yields the weak convergence of $\rho_{n}\left[u_{n, i}, R_{i} R_{j}\right] \rho u_{n, j}$ to $\rho\left[u_{i}, R_{i} R_{j}\right] \rho u_{j}$. Hence, we obtain

$$
-\overline{\rho \operatorname{div}(u)}+\rho \operatorname{div}(u)=\frac{1}{\mu+\xi}\left[\mu^{\prime}-\pi\right] .
$$

Now, we concentrate on the proof (see also [6]) of the strong convergences stated in theorem 3.2. From the equality $s=\bar{s}$ and the fact that $\rho \log \rho$ is strictly convex, we deduce easily the strong convergence 
of $\rho_{n}$ to $\rho$ in $L^{p}((0, T) \times \Omega)$, using Young measures. Hence, to get the convergence of $\rho_{n}$ to $\rho$ in $C\left([0, T] ; L^{p}(\Omega)\right)$ for $1 \leq p<+\infty$, we only need to show this for $p=1$. Then using appendix $C$ of [7], we see that $\rho_{n}$ and $\sqrt{\rho_{n}}$ are relatively compact in $C\left([0, T] ; L^{2}-w\right)$ and converge in this space respectively to $\sqrt{\rho}$ and to $\rho$. In particular we have $\rho(0)=\rho^{0}$. Next to get the strong convergence of $\sqrt{\rho_{n}}$ to $\sqrt{\rho}$ in $C\left([0, T] ; L^{2}\right)$ we notice that for any sequence $t_{n}$ of $[0, T]$ converging to $t$ we have

$$
\int_{\Omega} \sqrt{ } \rho_{n}{ }^{2}\left(t_{n}\right)=\int_{\Omega} \rho_{n}^{0} \rightarrow \int_{\Omega} \rho^{0}=\int_{\Omega} \sqrt{ } \rho^{2}(t) .
$$

Hence $\rho_{n}$ converges in $C\left([0, T] ; L^{1}\right)$ to $\rho$.

Next, to get the convergence of $\rho_{n} u_{n}$ to $\rho u$ in $L^{p}\left(0, T ; L^{r}\right)$. We use the bound of $\rho_{n} u_{n}$ in $L^{\infty}\left(0, T ; L^{2}\right)$ as well as the following strong convergences

$$
\begin{gathered}
\sqrt{\rho_{n}} \rightarrow \sqrt{\rho} \quad \text { in } \quad L^{p}((0, T) \times \Omega) . \\
\sqrt{\rho_{n}} u_{n} \rightarrow \sqrt{\rho} u \quad \text { in } \quad L^{2}((0, T) \times \Omega) .
\end{gathered}
$$

For this last convergence we use that $\rho_{n}\left|u_{n}\right|^{2}$ converges weakly to $\rho|u|^{2}$ and that $\sqrt{\rho_{n}} u_{n}$ converges weakly to $\sqrt{\rho} u$ (this can be deduced from lemma comp in the same way as the weak convergence of $\rho_{n} u_{n}$ ).

Moreover, using the bound on $D u_{n}$ in $L^{2}$ we deduce a bound on $\rho u$ in $L^{p}\left(0 . T ; L^{r}\right)$ for any $p>2$ and $2 \leq r \leq \frac{2 p N}{p N-1}$ if $N \geq 3$ ( $2 \leq r<\frac{2 p N}{p N-4}$ if $N=2$ and the bound is local in space if $\Omega=\mathbb{R}^{2}$ ). This yields the convergence stated in the remark.

The convergence of $\rho_{n} u_{n} \otimes u_{n}$ to $\rho u \otimes u$ is deduced easily from the convergence of $\sqrt{\rho_{n}} u_{n}$ to $\sqrt{\rho} u$ in $L^{p}\left(0 . T: L^{r}\right)$ for some couple $(p, r)$ with $p>2$ and $r>2$.

\section{EXISTENCE RESULTS}

In this section we are going to prove the existence of weak solutions $(\rho, u, \pi)$. It is classical (see for instance [6]) to deduce the existence of solutions, using the compactness results already shown, via a regularization (or some layers of regularization) of the equations or via a time discretization, which uses stationary problems. Nevertheless we are not going to use this classical method but we are going to show a more general convergence result and deduce the existence of solutions to our system as 
a consequence of that result. Besides, one of our motivations to study the system (1)-(4) is the following convergence result concerning solutions of the compressible Navier-Stokes equations as $\gamma$ tend to the infinity.

Let $\gamma_{n}$ be a sequence of nonnegative real numbers that goes to infinity. Let $\left(\rho_{n}, u_{n}\right)$ be a sequence of solutions of the isentropic compressible Navier-Stokes equations

$$
\left\{\begin{array}{l}
\frac{\partial \rho}{\partial t}+\operatorname{div}(\rho u)=0, \quad \rho \geq 0, \\
\frac{\partial \rho u}{\partial t}+\operatorname{div}(\rho u \otimes u)-\mu_{n} \Delta u-\xi_{n} \nabla \operatorname{div} u+a \nabla \rho^{\gamma_{n}}=0 .
\end{array}\right.
$$

where $\mu_{n}>0$ and $\mu_{n}+\xi_{n}>0, \mu_{n}$ and $\xi_{n}$ tend respectively to $\mu$ and $\xi$ as $n$ goes to the infinity, with $\mu>0$ and $\mu+\xi>0$ (in the sequel, we assume for simplicity that $\mu_{n}=\mu$ and $\xi_{n}=\xi$ ). Global weak solutions of the above system have been shown to exist by P.-L. Lions ([8], [9]), if we assume in addition that $\gamma_{n}>\frac{N}{2}$ if $N \geq 4, \gamma_{n} \geq \frac{3}{2}$ if $N=2$ and $\gamma_{n} \geq \frac{9}{5}$ if $N=3$. These assumptions are true for $n$ large enough. The sequence $\left(\rho_{n}, u_{n}\right)$ satisfies in addition the following initial conditions and the following bounds,

$$
\left.\rho_{n} u_{n}\right|_{t=0}=m_{n}^{0},\left.\rho_{n}\right|_{t=0}=\rho_{n}^{0}
$$

where $0 \leq \rho_{n}^{0}$ a.e., $\rho_{n}^{0}$ is bounded in $L^{1}(\Omega)$ and $\rho_{n}^{0} \in L^{\gamma_{n}}$ with $\int\left(\rho_{n}^{0}\right)^{\gamma_{n}} \leq C \gamma_{n}$ for some fixed $C, m_{n}^{0} \in L^{2 \gamma_{n} /\left(\gamma_{n}+1\right)}(\Omega)$, and $\rho_{n}^{0}\left|u_{n}^{0}\right|^{2}$ is bounded in $L^{1}$, denoting by $u_{n}^{0}=\frac{m_{n}^{0}}{\rho_{n}^{0}}$ on $\left\{\rho_{n}^{0}>0\right\}, u_{n}^{0}=0$ on $\left\{\rho_{n}^{0}=0\right\}$. In the case of $\mathbf{T}^{\mathrm{N}}$, we also assume that $f \rho_{n}^{0}=M_{n}$, for some $M_{n}$ such that $0<M_{n} \leq M<1$ and $M_{n} \rightarrow M$. Furthermore, we assume that $\rho_{n}^{0} u_{n}^{0}$ converges weakly in $L^{2}$ to some $m^{0}$ and that $\rho_{n}^{0}$ converges weakly in $L^{1}$ to some $\rho^{0}$. Our last requirement concerns the following energy bounds we impose on the sequence of solutions we consider,

$$
E_{n}(t)+\int_{0}^{t} D_{n}(s) d s \leq E_{n}^{0} \quad \text { a.e. } t, \frac{d E_{n}}{d t}+D_{n} \leq 0 \quad \text { in } \quad \mathcal{D}^{\prime}(0, \infty)
$$

where $E_{n}(t)=\int \frac{1}{2} \rho_{n}\left|u_{n}\right|^{2}(t)+\frac{a}{\gamma_{n}-1}\left(\rho_{n}\right)^{\gamma_{n}}(t), D_{n}(t)=\int \mu\left|D u_{n}\right|^{2}(t)$ $+\xi\left(\operatorname{div} u_{n}\right)^{2}(t)$ and $E_{n}^{0}=\int \frac{1}{2} \rho_{n}^{0}\left|u_{n}^{0}\right|^{2}+\frac{a}{\gamma_{n}-1}\left(\rho_{n}^{0}\right)^{\gamma_{n}}$. 
We recall that the results in [6] yield the existence of solutions $\left(\rho_{n}, u_{n}\right)$ satisfying the above requirements if we have $\gamma_{n}>\frac{N}{2}$ if $N \geq 4, \gamma_{n} \geq \frac{3}{2}$ if $N=2$ and $\gamma_{n} \geq \frac{9}{5}$ if $N=3$. We wish to mention an additional estimate which is available but that, however, we shall not use in this proof. Indeed, the proof made in P.-L. Lions [6] (Chapter 7, section 7.1) yields the following bound for all $T \in(0, \infty)$

$$
\int_{0}^{T} d t \int \rho_{n}^{\gamma_{n}+\theta_{n}} \leq C \gamma_{n} \text { where } \theta_{n}=\frac{2}{N} \gamma_{n}-1 .
$$

Unfortunately, this estimate is not uniform in $n$. Instead, we shall use another estimate which can be derived as (57) was in [6] and which is uniform in $n$, namely an $L^{1}$ bound for $\left(\rho_{n}\right)^{\gamma_{n}}$.

Without loss of generality, extracting subsequences if necessary, we can assume that $\left(\rho_{n}, u_{n}\right)$ converges weakly to $(\rho, u)$. More precisely we can assume that $\rho_{n} \rightarrow \rho$ weakly in $L^{p}((0, T) \times \Omega)$ for any $1 \leq p \leq \infty$ and that $\rho \in L^{\infty}\left(0, T ; L^{p}\right)$ (in fact we will show that $\rho$ actually satisfies $0 \leq \rho \leq 1$ ), $u_{n} \rightarrow u$ weakly in $L^{2}\left(0, T ; H_{l o c}^{1}\right)$.

THEOREM 4.1. - Under the above conditions, we have $0 \leq \rho \leq 1$ and

$$
\left(\rho_{n}-1\right)_{+} \rightarrow 0 \quad \text { in } L^{\infty}\left(0, T ; L^{p}\right) \text { for any } 1 \leq p<+\infty \text {. }
$$

Moreover, $\left(\rho_{n}\right)^{\gamma_{n}}$ is bounded in $L^{1}$ (for $n$ such that $\gamma_{n} \geq N$ ). Then extracting subsequences again, there exists $\pi \in \mathcal{M}((0, T) \times \Omega)$ such that

$$
\left(\rho_{n}\right)^{\gamma /} \underset{n}{\longrightarrow} \pi
$$

If in addition $\rho_{n}^{0}$ converges in $L^{1}$ to $\rho^{0}$ then $(\rho, u, \pi)$ is a solution of (1)-(4) and the following strong convergences hold

$$
\begin{gathered}
\rho_{n} \rightarrow \rho \quad \text { in } C\left(0, T ; L^{p}(\Omega)\right) \text { for any } 1 \leq p<+\infty \\
\rho_{n} u_{n} \rightarrow \rho u \quad \text { in } \quad L^{p}\left(0, T ; L^{q}(\Omega)\right) \text { for any } 1 \leq p<+\infty, 1 \leq q<2 \\
\rho_{n} u_{n} \otimes u_{n} \rightarrow \rho u \otimes u \quad \text { in } \quad L^{p}\left(0, T ; L^{1}(\Omega)\right) \text { for any } 1 \leq p<+\infty .
\end{gathered}
$$

Remark 1. - The limit $(\rho, u, \pi)$ satisfies in addition the following energy bounds which is to be compared with (17)

$$
\left\{\begin{array}{l}
E(t)+\int_{0}^{t} D(s) d s \leq E^{0}+\liminf _{n} \frac{\left(\rho_{n}^{0}\right)^{\gamma_{n}}}{\gamma_{n}} \text { a.e. } t \\
\frac{d E}{d t}+D \leq 0 \text { in } \mathcal{D}^{\prime}(0, \infty)
\end{array}\right.
$$


where $E$ and $D$ are defined as in (17).

Remark 2. - Theorem 4.1 yields immediately the existence theorem existence. In fact we can take, for instance, for any $n>\sup \left(\frac{N}{2} \cdot 2\right)$, $\gamma_{n}=n, \rho_{n}^{0}=\rho^{0}$ and $m_{n}^{0}=m^{0}$, we see then that

$$
\liminf _{n} \frac{\left(\rho_{n}^{0}\right)^{\gamma_{n}}}{\gamma_{n}}=0
$$

and hence (17) is satisfied.

Proof. - The proof is divided in three steps.

Step 1. - From the energy conservation and the mass conservation, we deduce that for any $1<p<\infty$, we have for $n$ such that $\gamma_{n}>p$,

$$
\begin{aligned}
\left\|\rho_{n}\right\|_{L^{\infty}\left(0, T ; L^{p}\right)} & \leq\left\|\rho_{n}\right\|_{L^{\infty}\left(0, T^{\prime}: L^{1}\right)}^{\theta_{n}}\left\|\rho_{n}\right\|_{L^{\infty}\left(0, T: L^{-n_{n}}\right)}^{1-\theta_{n}} \\
& \leq M_{n}^{\theta_{n}}\left(C \gamma_{n}\right)^{\left(1-\theta_{n}\right) / \gamma_{n}}
\end{aligned}
$$

where we have used Holder's inequality and where $\theta_{n}$ is given for any $n$ by $\frac{1}{p}=\theta_{n}+\frac{1-\theta_{n}}{\gamma_{n}}$. Then, letting $n$ go to infinity, we deduce that $\theta_{n} \rightarrow \frac{1}{p}$ and that

$$
\|\rho\|_{L^{\infty}\left(0, T ; L^{p}\right)} \leq \liminf _{n \rightarrow \infty}\left\|\rho_{n}\right\|_{L^{\infty}\left(0, T ; L^{p}\right)} \leq M^{\frac{1}{n^{n}}}
$$

Hence, letting $p$ go to infinity, we obtain

$$
\|\rho\|_{L^{\infty}\left(0, T ; L^{\infty}\right)} \leq \liminf _{p \rightarrow \infty}\|\rho\|_{L^{\infty}\left(0, T ; L^{p}\right)} \leq 1
$$

We next introduce $\phi_{n}=\left(\rho_{n}-1\right)_{+}$. We are going to show that $\phi_{n}$ goes to 0 uniformly in $t$ in all $L^{p}$ spaces. In fact, from the energy conservation that we have for any $t$, we deduce

$$
\int_{\Omega}\left(1+\phi_{n}\right)^{\gamma / n} 1_{\left\{\phi_{n}>0\right\}} \leq \int_{\Omega}\left(\rho_{n}\right)^{\gamma_{n}} \leq C \gamma_{n} .
$$

Next, for any $p>1$, there exists a constant $a$ (for instance we can take $a_{p}=\frac{1}{2 p !}$ if $p$ is an integer) such that for any $k$ large enough the following inequality holds,

$$
(1+x)^{k} \geq 1+a_{p} k^{p} x^{p}
$$


for any nonnegative $x$. To see that we can consider the case where $p$ is an integer and then make an "interpolation". In fact if $p \in I N$, we can take $f(x)=(1+x)^{k}-a_{p} k^{p} x^{p}$ and notice that a sufficient condition for $f$ to be nonnegative on $\mathbb{R}^{+}$is that $f^{(p)}(x)=k(k-1) \ldots(k-p+1)(1+x)^{k-p}-$ $a_{p} p ! k^{p}$ is nonnegative in $\mathbb{R}^{+}$and we can see that this holds true for $k$ large enough if $a=\frac{1}{2 p !}$ for instance. Next, if $p<q \leq p+1$ with $p \in \mathbb{N}$, we have $(1+x)^{k} \geq 1+a_{p}(k x)^{p}$ and $(1+x)^{k} \geq 1+a_{p+1}(k x)^{p+1}$ for $k$ large enough. Then, we notice that for all $x \in \mathbb{R}^{+}$we have $(k x)^{q} \leq \sup \left((k x)^{p},(k x)^{p+1}\right)$, hence we can take $a_{q}=\inf \left(a_{p}, a_{p+1}\right)=a_{p+1}$.

Hence, we have for $n$ large enough

$$
\int_{\Omega} \phi_{n}^{p} \leq \frac{C a_{p}}{\left(\gamma_{n}\right)^{p-1}}
$$

which yields the convergence of $\left(\rho_{n}-1\right)_{+}$to 0 . Let us notice that this convergence is obviously "very fast".

Step 2. - Now, we turn to the proof of the $L^{1}$ bound on $\left(\rho_{n}\right)^{\gamma_{n}}$. We begin by treating the whole space case and then explain the necessary modifications we in the periodic case. Applying as in [6], the operator $(-\Delta)^{-1}$ div to $(54)$, we obtain

$$
\left(\rho_{n}\right)^{\gamma_{n}}=\partial_{t}(-\Delta)^{-1} \operatorname{div}\left(\rho_{n} u_{n}\right)-R_{i} R_{j}\left(\rho\left(u_{n}\right)_{i}\left(u_{n}\right)_{j}\right)+(\mu+\xi) \operatorname{div}\left(u_{n}\right) .
$$

Then multiplying (60) by $\rho_{n}$, we deduce (we omit the indices $n$ in the right hand side for the sake of clarity),

$$
\left\{\begin{aligned}
\left(\rho_{n}\right)^{\gamma_{n}+1} & =\partial_{t}\left[\rho(-\Delta)^{-1} \operatorname{div}(\rho u)\right]+\operatorname{div}\left[\rho u(-\Delta)^{-1} \operatorname{div}(\rho u)\right] \\
& +\rho u_{i} R_{i} R_{j}\left(\rho u_{j}\right)-\rho R_{i} R_{j}\left(\rho u_{i} u_{j}\right)+(\mu+\xi) \rho \operatorname{div}(u)
\end{aligned}\right.
$$

This manipulation and essentially the multiplication of $\rho_{n}$ by $\partial_{l}(-\Delta)^{-1} \operatorname{div}\left(\rho_{n} u_{n}\right)$ should be justified. This can be justified in the same way as for the product of $\rho$ by $\pi$. Then, integrating (61) over $(0, T) \times \mathbb{R}^{\mathrm{N}}$, we see that $\left(\rho_{n}\right)^{\gamma_{n}+1}$ is bounded in $L^{1}\left((0, T) \times \mathbb{R}^{\mathrm{N}}\right)$, uniformly in $\mathrm{n}$. In fact, the proof is the same as the proof of the bound of $\pi$ in $\mathcal{M}\left((0, T) \times \mathbb{R}^{\mathrm{N}}\right)$, so we are only going to mention the changes that must be performed. We also refer to [6] (Chapter 7, section 7.1) for the proof of (57). First, we notice that we do not have a $L^{\infty}$ bound on $\rho_{n}$. However, since $\int\left(\rho_{n}\right)^{\gamma_{n}}(t) \leq C \gamma_{n}$ for a.e. $t$, we see that there exists Vol. $16, n^{\circ} 3-1999$ 
a constant $C_{1}\left(\right.$ for instance $\left.C_{1}=\exp \left(\frac{C}{e}\right)\right)$, such that for any $n$, we have $\left\|\rho_{n}\right\|_{L^{\infty}\left(0, T ; L^{\gamma_{n}}\right)} \leq C_{1}$, since

$$
\sup _{\gamma>0}(C \gamma)^{1 / \gamma}=\exp \left(\frac{C}{e}\right)
$$

Next, we remark that the norm of $\rho_{n} u_{n}$ in $L^{\infty}\left(0, T ; L^{2 \gamma_{n} /\left(\gamma_{n}+1\right)} \cap L^{1}\right)$ is independent of $n$ (we use here the fact that the norm of $\sqrt{\rho_{n}}$ in $L^{\infty}\left(L^{2 \gamma_{n}} \cap L^{2}\right)$ is bounded independently of $n$ and that $\sqrt{\rho_{n}} u_{n}$ is bounded in $L^{\infty}\left(L^{2}\right)$ ). Hence, we may write

$$
\left|\int_{0}^{T} \int_{\mathbb{R}^{N}} \partial_{t}\left[\rho(-\Delta)^{-1} \operatorname{div}(\rho u)\right]\right| \leq 2\|\rho\|_{L^{\infty}\left(L^{q^{\prime}}\right)}\|\rho u\|_{L^{\infty}\left(L^{\prime \prime}\right)}
$$

with $\left(\frac{1}{r}-\frac{1}{N}\right)+\frac{1}{q^{\prime}}=1,1 \leq r \leq \frac{2 \gamma_{n}}{\gamma_{n}+1}$ and $1 \leq q^{\prime} \leq \gamma_{n}$ (such a choice is possible if $\gamma_{n} \geq N$ for instance).

For the second term, we only need to show that $U=$ $\rho_{n} u_{n}(-\Delta)^{-1} \operatorname{div}\left(\rho_{n} u_{n}\right)$ belongs to $L^{\infty}\left(L^{1}\right)$ for all $n$, which can be deduced from the following bound

$$
\|U\|_{L^{\infty}\left(L^{1}\right)} \leq\left\|\rho_{n} u_{n}\right\|_{L^{\infty}\left(L^{1}\right)}^{2},
$$

with $q=\frac{2 N}{N+1} \leq \frac{2 \gamma_{n}}{\gamma_{n}+1}$, since $\gamma_{n} \geq N$.

For the third and the fourth term, we must distinguish two cases, namely $N \geq 3$ and $N=2$. In the first case, we have $\rho|u|^{2} \in L^{1}\left(L^{s}\right)$, with $\frac{1}{s}=\frac{N-2}{N}+\frac{1}{\gamma_{n}}<1$. Therefore $R_{i} R_{j}\left(\rho u_{i} u_{j}\right) \in L^{1}\left(L^{s}\right)$ and hence $\rho R_{i} R_{j}\left(\rho u_{i} u_{j}\right) \in L^{1}\left(L^{1}\right)$, since $\frac{N-2}{N}+2 \frac{1}{\gamma_{n}} \leq 1$. For the third term we recall the fact that $\rho_{n} u_{n}, R_{i} R_{j}\left(\rho\left(u_{n}\right)_{i}\left(u_{n}\right)_{j}\right) \in L^{2}\left(L^{r} \cap L^{1}\right)$, where $\frac{1}{r}=\frac{N-2}{2 N}+\frac{1}{\gamma_{n}} \geq 2$. The case $N=2$ is treated using the bound on $u_{n}$ in $L^{2}(0, T ; B M O)$ and on $\rho_{n} u_{n}$ in $L^{2}\left(L^{3 / 2}\right)$ (if $\gamma_{n} \geq 3$ ), which yield

$$
\left\|\left[u, R_{i} R_{j}\right]\left(\rho u_{j}\right)\right\|_{L^{1}\left(0, T ; L^{3 / 2}\right)} \leq C\|u\|_{L^{2}(0, T ; B M O)}\|\rho u\|_{L^{2}\left(0, T ; L^{3 / 2}\right)}
$$

and, multiplying by $\rho$, we deduce

$$
\begin{aligned}
& \left\|\rho\left[u, R_{i} R_{j}\right]\left(\rho u_{j}\right)\right\|_{L^{1}\left(0, T ; L^{1}\right)} \\
& \quad \leq C\|\rho\|_{L^{\infty}\left(0, T ; L^{3}\right)}\|u\|_{L^{2}(0, T ; B M O)}\|\rho u\|_{L^{2}\left(0, T ; L^{3 / 2}\right)} .
\end{aligned}
$$


Finally for the fifth term, we have the following straightforward computation

$$
\mid \int_{0}^{T} \int_{\mathbb{R}^{N}} \rho_{n} \text { div } u_{n} \mid \leq\left\|\rho_{n}\right\|_{L^{2}\left(0, T ; L^{2}\right)} \| \text { div } u_{n} \|_{L^{2}\left(0, T ; L^{2}\right)} .
$$

where we use the fact that $\rho_{n}$ is bounded in $L^{2}\left(0, T ; L^{2}\right)$ since $\gamma_{n} \geq N \geq 2$.

Using this bound on $\left(\rho_{n}\right)^{\gamma_{n}+1}$ and the fact that $\rho \in L^{\infty}\left(0, T ; L^{1}\right)$, we deduce the desired bound

$$
\int_{0}^{T} \int_{\mathbb{R}^{\mathrm{N}}}\left(\rho_{n}\right)^{\gamma_{n}} \leq \int_{0}^{T} \int_{\mathbb{R}^{\mathrm{N}}}\left(\rho_{n}\right)^{\gamma_{n}+1}+\rho_{n}
$$

Hence extracting subsequences again, there exists $\pi \in \mathcal{M}((0, T) \times \Omega)$ such that

$$
\left(\rho_{n}\right)^{\gamma_{n}} \underset{n}{\longrightarrow} \pi
$$

Step 3. - Finally, we show that $(\rho, u, \pi)$ is actually a solution of the initial system and that the strong convergences hold. First, we observe that $\frac{\partial \rho_{n}}{\partial t}$ is bounded in $L^{\infty}\left(0, T ; W^{-1,1}\right)$ and that $u_{n}$ is bounded in $L^{2}\left(0, T ; H^{1}\right)$. Then, using the compactness lemma 3.3, we get that $\rho_{n} u_{n}$ converges weakly to $\rho u$. On the other hand, using the bound on $\left(\rho_{n}\right)^{\gamma_{n}}$ in $L^{1}$, we deduce that $\frac{\partial \rho_{n} u_{n}}{\partial t}$ is bounded in $L^{1}\left(0, T ; W^{-1,1}\right)$ and hence $\rho_{n} u_{n} \otimes u_{n} \rightarrow \rho u \otimes u$. The only point that should be proved is the relation $\rho \pi=\pi$. Using the same notations as in the previous section $(s=\rho \log (\rho)$ and $\bar{s}=\bar{\rho} \log (\rho))$, we get

$$
\frac{\partial}{\partial t}(\bar{s}-s)+\operatorname{div}[(\bar{s}-s) u]=-\overline{\rho \operatorname{div} u}+\rho \operatorname{div} u .
$$

Then applying the operator $(-\Delta)^{-1}$ div to the momentum equation, multiplying by $\rho_{n}$ and passing to the limit (extracting subsequences if necessary), we obtain

$$
\left\{\begin{aligned}
\overline{\left(\rho_{n}\right)^{\gamma_{n}+1}}-(\mu+\xi) \overline{\rho \operatorname{div}(u)} & =\partial_{t} \overline{\left[\rho(-\Delta)^{-1} \operatorname{div}(\rho u)\right]} \\
& +\operatorname{div} \overline{\left[\rho u(-\Delta)^{-1} \operatorname{div}(\rho u)\right]} \\
& +\overline{\rho u_{i} R_{i} R_{j}\left(\rho u_{j}\right)-\rho R_{i} R_{j}\left(\rho u_{i} u_{j}\right)}
\end{aligned}\right.
$$

Changing the order of the multiplication by $\rho$ and the passage to the weak limit, we obtain

$$
\left\{\begin{aligned}
\rho \pi-(\mu+\xi) \rho \operatorname{div}(u) & =\partial_{t}\left[\rho(-\Delta)^{-1} \operatorname{div}(\rho u)\right] \\
& +\operatorname{div}\left[\rho u(-\Delta)^{-1} \operatorname{div}(\rho u)\right] \\
& +\rho u_{i} R_{i} R_{j}\left(\rho u_{j}\right)-\rho R_{i} R_{j}\left(\rho u_{i} u_{j}\right)
\end{aligned}\right.
$$


The same computations as in the last section show that the second hand sides of (64) and (65) are equal. Hence, we get

$$
-\overline{\rho \operatorname{div}(u)}+\rho \operatorname{div}(u)=\frac{1}{\mu+\xi}\left[\rho \pi-\overline{\left(\rho_{n}\right)^{m+1}}\right] .
$$

Reporting this in (63), we get

$$
\frac{\partial}{\partial t}(\bar{s}-s)+\operatorname{div}[(\bar{s}-s) u]=\frac{1}{\mu+\xi}\left[\rho \pi-\overline{\left(\rho_{n}\right)^{\gamma_{n}+1}}\right] .
$$

Next, we notice that $\rho \pi=\overline{\rho\left(\rho_{n}\right)^{\gamma_{n}}} \leq \overline{\left(\rho_{n}\right)^{\gamma_{n}+1}}$. Indeed we have

$$
\begin{aligned}
\overline{\left(\rho_{n}\right)^{\gamma_{n}+1}}-\overline{\rho_{n}} \overline{\left(\rho_{n}\right)^{\gamma_{n}}} & =\overline{\left(\left(\rho_{n}\right)^{\gamma_{n}}\right)\left(\rho_{n}-\rho\right)} \\
& =\overline{\left(\left(\rho_{n}\right)^{\gamma_{n}}-(\rho) \gamma_{n}\right)\left(\rho_{n}-\rho\right)} \\
& \geq 0
\end{aligned}
$$

where we have used that

$$
(\rho)^{r \prime \prime} \rightarrow 1_{\{\rho=1\}}
$$

almost everywhere and in $L^{p}((0, T) \times \Omega)$ for $1 \leq p<\infty$ which yields the following weak convergence

$$
(\rho)^{\gamma_{n}}\left(\rho_{n}-\rho\right)-0
$$

Next, integrating (66) in $x$, we get

$$
\frac{\partial}{\partial t} \int_{\Omega}(\bar{s}-s) d x \leq 0
$$

Then, since $\bar{s}-\left.s\right|_{t=0}=0$ and $s \leq \bar{s}$, we see that $s=\bar{s}$. Therefore, we obtain that

$$
\rho \pi=\overline{\left(\rho_{n}\right)^{\gamma_{n}+1}}
$$

Next, we see that for any $\varepsilon>0$ there exists $n_{0}$ such that for $n \geq n_{0}$ and $x \geq 0$, we have

$$
x^{\cdots+1} \geq x^{\gamma n}-\varepsilon
$$

Applying this inequality to $\rho_{n}$ and passing to the weak limit, we get

$$
\overline{\left(\rho_{n}\right)^{\gamma_{n}+1}} \geq \pi-\varepsilon \text {. }
$$


Then, letting $\varepsilon$ go to 0 , we get

$$
\rho \pi \geq \pi
$$

Next, using that $0 \leq \rho \leq 1$, we obtain

$$
\rho \pi \leq \rho
$$

However, since the product $\rho \pi$ is not defined almost everywhere, we must explain the above inequality. We denote by $\omega_{k}=k^{N+1} \omega(k$. $)$ a smooothing sequence in both variables $t$ and $x$, where $\omega \in C^{\infty}\left(\mathbb{R}^{N+1}\right), \omega \geq 0$, $f_{\mathbb{R}^{N+1}} \omega=1, \operatorname{Supp}(\omega) \in B_{1}\left(\mathbb{R}^{N+1}\right)$. Then, we denote by $\rho_{k}=\rho * \omega_{k}$ (resp $\pi_{k}=\pi * \omega_{k}$ ) a sequence of nonnegatif smooth functions converging to $p(\operatorname{resp} \pi)$

$$
\begin{cases}\rho_{k} \rightarrow \rho & \text { in } C\left([0, T] ; L^{p}\right) \cap C^{1}\left([0, T] ; H^{-1}\right), \\ \pi_{k} \rightarrow \pi & \text { in } W^{-1,2}\left(H^{1}\right)+L^{1}\left(L^{q}\right),\end{cases}
$$

for some $q>1$ and $p$ such that $\frac{1}{p}+\frac{1}{q}=1$. Hence writing $(\rho-1) \pi$ as

$$
(\rho-1) \pi=\left(\rho_{k}-1\right) \pi_{k}+\left(\rho-\rho_{k}\right) \pi_{k}+(\rho-1)\left(\pi-\pi_{k}\right)
$$

we conclude by letting $k$; go to the infinity.

Finally, we deduce that

$$
\rho \pi=\rho
$$

Therefore, $(\rho, u, \pi)$ is a solution of (1)-(4). The strong convergences stated in the theorem are then deduced easily as in the proof of the compactness theorem.

\section{GENERAL PRESSURE LAW AND INFINITE MASS}

In this section, we discuss two related issues. The first one is the case where we include a pressure $(\pi=p(\rho)$ on $\{\rho<1\})$, the fluid is assumed to behave as a general barotropic fluid as long as its density is smaller than 1. We also study the case where we no longer assume that the total mass is finite in the case of $\mathbb{R}^{\mathrm{N}}$. 
We begin with the case of a general pressure law fluid. The pressure law $p$ is assumed to be a continuous nondecreasing function on $[0, \infty)$ vanishing at 0 . For instance, we can take $p(\rho)=a \rho^{\gamma}$. Next, let $q$ be defined (up to a linear function) by $\frac{d}{d t}\left(\frac{q(t)}{t}\right)=\frac{p(t)}{t^{2}}$ for $t>0$. Notice then that multiplying the moment equation by $u$, the term $\pi$ div $u$ does not vanish and is equal to $p(\rho) \operatorname{div} u$, since $\operatorname{div} u=0$ on $\{\rho=1\}$. Then, using (1), we get taking $\beta(\rho)=q(\rho)$ in $(21)$

$$
\frac{\partial q(\rho)}{\partial t}+\operatorname{div}(q(\rho) u)=-p(\rho) \operatorname{div} u
$$

since $q(\rho)-\rho q^{\prime}(\rho)=-p(\rho)$. This equation need some justifications if $q(t)$ is not $C^{1}\left(q \in C^{1}([0, \infty))\right.$ if and only if $\left.\int_{0}^{1} \frac{p(t)}{t^{2}}<+\infty\right)$ and we only have to approximate $q$ by $q_{\delta}(\rho)=\rho \int_{c}^{\rho+\delta} \frac{p(t)}{t^{2}} \ldots$ Now, integrating (27), we obtain (at least formally)

$$
\frac{\partial}{\partial t} \int_{\Omega}\left[\rho \frac{|u|^{2}}{2}+q(\rho)\right]+\int_{\Omega} \mu(D u)^{2}+\xi(\operatorname{div} u)^{2}=\int_{\Omega} \rho u . f .
$$

Next since $q$ is bounded from below, we see that we deduce the same a priori bounds in the periodic case. We also notice that, in this case, we can make the computations directly on the term $p(\rho)$ div $u$, since $p(\rho)$ is bounded and that $\|p(\rho) \operatorname{div} u\|_{L^{1}} \leq \frac{C}{\varepsilon}+\varepsilon\|\operatorname{div} u\|_{L^{2}}$, for any $\varepsilon>0$.

In the whole space case, this bound from below is not sufficient to derive the desired bounds. We thus begin by the case when we can take $q$ such that $q \geq 0$. In fact if $\int \frac{p(t)}{t^{2}}<+\infty$ then we can take $q(\rho)=\rho \int_{0}^{\rho} \frac{p(t)}{t^{2}}$ and hence $q(\rho) \geq 0$. We then obtain (we omit here the force term $\mathrm{f}$ )

$$
\int_{\Omega} \rho \frac{|u|^{2}}{2}(T)+\int_{0}^{T} \int_{\Omega} \mu(D u)^{2}+\xi(\operatorname{div} u)^{2}=\int_{\Omega} \rho \frac{|u|^{2}}{2}(0)+q\left(\rho^{0}\right)
$$

and the same bounds follow. However if $\int \frac{p(t)}{t^{2}}=+\infty$, we can no longer take $q$ such that $q \geq 0$ and hence we cannot obtain such bounds directly. For instance, if $p(\rho)=a \rho^{\gamma}$ with $0<\gamma<1$, then $q(\rho)=-\frac{a}{1-\gamma} \rho^{\gamma}+C \rho$ and we can check easily that

$$
\sup _{\int \rho=M} \rho^{\gamma}=+\infty \text {. }
$$


One idea to recover some bounds is to introduce (as in P.-L. Lions [6]) a reference function $\bar{\rho}$, such that $0 \leq \bar{\rho} \leq 1, \bar{\rho} \in L^{1}$ and $\nabla\left(q^{\prime}(\bar{\rho})\right) \epsilon$ $L^{\infty}+L^{2}\left(\mathbb{R}^{N}\right)$. Another case, where we can recover bounds is the case when the force $\int$ is potential, namely $\int=-\nabla V$ for some $V$. In fact if the force is attractive enough we see that the fluid cannot disperse widely. We study below a case where we can recover some bounds, we assume that $V=V_{+}-V_{-}$, with $V_{+}, V_{-} \geq 0$ and

$$
\left\{\begin{array}{l}
p(\rho)=a \rho^{\gamma} \quad \text { with } \quad 0<\gamma<1 \\
V_{-} \in L^{1}+L^{\infty}, \\
\int_{\mathbb{R}^{N}}\left(V_{+}+1\right)^{-\frac{\gamma}{1-\gamma}}<+\infty \\
f=-\nabla V+f^{\prime}, \quad \text { where } f^{\prime} \text { satisfies }(19) .
\end{array}\right.
$$

In this case the energy estimates yield

$$
\frac{\partial}{\partial t} \int_{\Omega \Omega}\left[\rho \frac{|u|^{2}}{2}+q(\rho)+\rho V\right]+\int_{\Omega \Omega} \mu(D u)^{2}+\xi(\operatorname{div} u)^{2}=\int_{\Omega} \rho u . f^{\prime}
$$

To recover some bounds, we must show that

$$
\inf _{\rho \rho=M, 0 \leq \rho \leq 1} \int \rho V+q(\rho)>-\infty .
$$

In fact, we have

$$
\int \rho V_{-} \leq(1+M)\left\|V_{-}\right\|_{L^{1}+L^{\infty}}
$$

and the infimum of $\rho V_{+}-\frac{a}{1-\gamma} \rho^{\gamma}$ under the constraint $\int \rho=M$ is reached for

$$
\bar{\rho}=\left(\frac{1-\gamma}{a} V_{+}+C\right)^{-\frac{1}{1-\gamma}}
$$

where $C$ is such that $\int \bar{\rho}=M$. Hence, for almost all $t$, we have

$$
\int \rho V_{+}+q(\rho) \geq \int \bar{\rho} V_{+}+q(\bar{\rho}) \geq-\infty
$$

and we recover the desired bounds as above.

We next turn to the study of another topic, namely the case of infinite mass in the whole space case. We require $\rho^{0}$ to satisfy the following conditions

$$
\left\{\begin{array}{l}
\rho-\bar{\rho} \in L^{2}\left(\mathbb{R}^{\mathrm{N}}\right) \quad \text { where } \bar{\rho} \text { satisfies } \\
0 \leq \bar{\rho} \leq 1, \quad \text { meas }\{\bar{\rho}=1\} \text { is finite, } \\
\nabla\left(q^{\prime}(\bar{\rho})\right) \in L^{\infty}+L^{2}\left(\mathbb{R}^{\mathrm{N}}\right) \quad \text { and } \quad \mathrm{q}^{\prime \prime}(\bar{\rho}) \geq \mathrm{c}
\end{array}\right.
$$


for some constant $c>0$. Notice that we impose no integrability conditions on $\bar{\rho}$. In this case the energy estimates are the same as in the case where we only include a pressure. In fact, since $\rho$ and $\bar{\rho}$ are bounded and $q^{\prime \prime}(\bar{\rho}) \geq c$. we can find two constants $c_{1}$ and $c_{2}$, such that we have

$$
c_{1}(\rho-\bar{\rho})^{2} \geq q(\rho)-q(\bar{\rho})-q^{\prime}(\bar{\rho})(\rho-\bar{\rho}) \geq c_{2}(\rho-\bar{\rho})^{2} .
$$

We begin our investigations by the following simpler case $(\bar{\rho}$ is a constant such that $0 \leq \bar{\rho}<1$ ). The energy estimates then yields the following inequality

$$
\int_{\Omega} \rho \frac{|u|^{2}}{2}+c_{2}(\rho-\bar{\rho})^{2}(T)+\int_{0}^{T} \int_{\Omega} \nu(D u)^{2} \leq \int_{\Omega} \rho \frac{|u|^{2}}{2}(0)+c_{1}\left(\rho^{0}-\bar{\rho}\right)^{2}
$$

Next in order to deduce a bound for $\pi$ in $L^{1}$, we multiply (37) by $\rho-\bar{\mu}$, and we obtain

$$
\begin{aligned}
(1-\bar{\rho}) \pi & =\partial_{+}\left[(\rho-\bar{\rho})(-\Delta)^{-1} \operatorname{div}(\rho u)\right]+\operatorname{div}\left[\rho u(-\Delta)^{-1} \operatorname{div}(\rho u)\right] \\
& +\rho u_{i} R_{i} R_{j}\left(\rho u_{j}\right)-(\rho-\bar{\rho}) R_{i} R_{j}\left(\rho u_{i} u_{j}\right)+(\mu+\xi)(\rho-\bar{\rho}) \operatorname{din}(u)
\end{aligned}
$$

Then we deduce a bound on $\pi$ in $L^{1}$ integrating this equality. In fact, all the terms in the right-hand side are treated as in section 2 , replacing $\rho$ by $\rho-\bar{\rho}$ (recall that $\rho-\bar{\rho} \in L^{\infty}\left(0, T ; L^{2}\left(\mathbb{R}^{N}\right)\right)$ ). The case $N=2$ does not create further difficulty and we treat the term $(\rho-\bar{\rho}) R_{i} R_{j}\left(\rho u_{i} u_{j}\right)$ as follows

$$
(\rho-\bar{\rho}) R_{i} R_{j}\left(\rho u_{i} u_{j}\right)=(\rho-\bar{\rho})\left[R_{i} R_{j}, u_{i}\right]\left(\rho u_{j}\right)+(\rho-\bar{\rho}) u_{i} R_{i} R_{j}\left(\rho u_{j}\right) .
$$

In the general case, namely when $\bar{\rho}$ is no longer assumed to be a constant, the energy estimates yield the same bounds as above. In fact, for the extra term, we have

$$
\left|\int \rho u \nabla\left(q^{\prime}(\bar{\rho})\right)\right| \leq\|\rho u\|_{L^{1} \cap L^{2}}\left\|\nabla\left(q^{\prime}(\bar{\rho})\right)\right\|_{L^{*}+L^{2}} .
$$

Next, in order to obtain the estimate on $\pi$, we use the existence of an $\alpha>0$ such that meas $\{\bar{\rho}>1-\alpha\}$ is finite. In fact

$$
\cap_{\alpha>0}\{\bar{\rho}>1-\alpha\}=\{\bar{\rho}=1\} \text {. }
$$

Using this $\alpha$, we see that

$$
\bar{\rho}-\inf (\bar{\rho}, 1-\alpha) \in L^{2}
$$

then multiplying (37) by $\rho-\inf (\bar{\rho}, 1-\alpha)$ instead of $\rho-\bar{\rho}$, we deduce

$$
\alpha \int_{\mathbb{R}^{*}} \pi \leq \int[1-\inf (\bar{\rho}, 1-\alpha)] \pi \text {. }
$$

which yields the same $L^{1}$ bound since $\rho-\inf (\bar{\rho} .1-\alpha) \in L^{\infty}\left(L^{2}\right)$. 


\section{CONVERGENCE TO THE INCOMPRESSIBLE NAVIER-STOKES SYSTEM}

In this section, we discuss two results concerning the convergence to the incompressible Navier-Stokes system in the case of two space dimensions $(\mathrm{N}=2)$. These two results are still true, for $N \geq 3$, on any interval of time $(0, T)$ for which we know that there exists a strong solution $u$ (which is unique) for the incompressible Navier-Stokes equation.

The first issue concerns the limit $M \rightarrow 1$ (in the periodic case). Let $M_{n}$ be a sequence of real numbers $\left(0<M_{n}<1\right)$ converging to 1 and $\left(p_{n}, u_{n}, \pi_{n}\right)$ a sequence of solutions of (1) - (4) in $\mathbf{T}^{2}$ satisfying the energy inequality. Let us explain the heuristics which lead to the incompressible Navier-Stokes equation. Indeed since $\rho_{n} \leq 1$ and $f \rho_{n}(t)=M_{n} \rightarrow 1$ we deduce that $\rho \rightarrow 1$. Nexl, we conclude from (1) that the weak limit of $u_{n}$ satisfies the divergence-free condition : div $u=0$. Hence, we expect that $\left(\rho_{n}, u_{n}, \pi_{n}\right)$ converges, in some sense, to $(1, u, \pi)$, where $(u, \pi)$ is the weak solution of the incompressible Navier-Stokes equations

$$
\frac{\partial u}{\partial t}+\operatorname{div}(u \otimes u)-\mu \Delta u+\nabla \pi=0 .
$$

We shall show that this heuristic derivation is basically correct if we impose in addition some extra conditions on the initial data. We assume that $\left(\rho_{n}, u_{n}, \pi_{n}\right)$ satisfy the following initial conditions

$$
\left.\rho_{n} u_{n}\right|_{t-0}=m_{n}^{0},\left.\rho_{n}\right|_{t-0}=\rho_{n}^{0},
$$

where $0 \leq \rho_{n}^{0} \leq 1$ a.e., $f \rho_{n}^{0}=M_{n}$, and $\sqrt{\rho_{n}^{0}} u_{n}^{0}$ converges strongly in $L^{2}$ to a divergence-free vector $u^{0}$.

THEOREM 6.1. - Under the above assumptions, $\rho_{n}$ converges to 1 in $C\left([0, T] ; L^{p}(\Omega)\right)$ for $1 \leq p<+\infty, \sqrt{\rho_{n}} u_{n}$ converges to $u$ in $L^{\infty}\left(0, T ; L^{2}(\Omega)\right)$ and $D u_{n}$ converges to $D u$ in $L^{2}\left(0, T ; L^{2}(\Omega)\right)$ for all $T \in(0, \infty)$ where $u$ is the unique solution of the incompressible NavierStokes equations corresponding to the initial condition $u^{0}$.

Proof. - The proof of this theorem is similar to a proof introduced in [11]. First it is easy to see that $\rho_{n}$ converges to 1 in $C\left([0, T] ; L^{p}\right.$ ) (for $1 \leq p<+\infty)$. Next, we can assume, extracting subsequences if necessary, that $u_{n}$ converges weakly in $L^{2}\left(0, T ; H^{1}(\Omega)\right)$ to some $v$. Then, applying lemma 3.3, we deduce that $\sqrt{\rho_{n}} u_{n}$ and $\rho_{n} u_{n}$ converge weakly-star to $v$ in $L^{\infty}\left(0, T ; L^{2}(\Omega)\right)$ and hence $v$ is divergence free. At this point, we cannot 
deduce, as before, the weak convergence of $\rho_{n} u_{n} \otimes u_{n}$ to $v \otimes v$ since we have not a uniform bound on $\pi_{n}$ and hence no compactness in time for $\rho_{u} u_{n}$.

Let $u_{0}^{\delta} \in C_{0}^{\infty}(\Omega)$ be such that $: \operatorname{div} u_{0}^{\delta}=0$ in $\Omega, u_{0}^{\delta} \rightarrow u^{0}$ in $L^{2}(\Omega)$ as $\delta$ goes to $0_{+}$. We denote by $u^{\delta}$ the solution of the incompressible Navier-Stokes equations corresponding to the initial condition $u_{0}^{\delta}$. As is well-known, $u^{\delta}$ is smooth on $([0, \infty) \times \Omega)$ and $u^{\delta}$ converges to $u$ in $L^{2}\left(0, T ; H^{1}(\Omega)\right) \cap C\left([0, T] ; L^{2}(\Omega)\right)$ (and thus in $L^{4}(\Omega \times(0, T))$ for all $T \in(0, \infty)$. The energy inequality for $u_{n}$ yields for almost all $t$

$$
\int_{\Omega} \frac{1}{2} \rho_{n}\left|u_{n}\right|^{2}(t)+\int_{0}^{t} \int_{\Omega} \mu\left|D u_{n}\right|^{2}+\xi\left(\text { div } u_{n}\right)^{2} \leq \int_{\Omega} \frac{1}{2} \rho_{n}^{0}\left|u_{n}^{0}\right|^{2} .
$$

Then using the conservation of energy for $u^{t}$, we get for all $t$

$$
\int_{\Omega} \frac{1}{2}\left|u^{\delta}\right|^{2}(t)+\int_{0}^{t} \int_{\Omega} \mu\left|D u^{\delta}\right|^{2}(s) d s=\int_{\Omega} \frac{1}{2}\left|u_{0}^{\delta}\right|^{2}
$$

In addition, since $u^{\delta}$ is divergence-free and $P\left(\rho_{n} u_{n}\right)$ is continuous in time, we get for all $t$

$$
\left\{\begin{array}{l}
\int_{\Omega} \rho_{n} u_{n} \cdot u^{\delta}(t)-\int_{0}^{t} \int_{\Omega} \rho_{n} u_{n} \cdot \partial_{t} u^{\delta}+\rho_{n} u_{n} u_{n} \cdot \nabla u^{\delta}+ \\
+2 \mu \int_{0}^{t} \int_{\Omega} \nabla u_{n} . \nabla u^{\delta}+\mu \int_{0}^{t} \int_{\Omega} u_{n} \Delta u^{\delta}=\int_{\Omega} \rho_{n}^{0} u_{n}^{0} \cdot u_{0}^{\delta} .
\end{array}\right.
$$

Combining (77), (78) and (79), we get

$$
(80)\left\{\begin{array}{l}
\int_{\Omega} \frac{1}{2}\left|\sqrt{\rho_{n}} u_{n}-u^{\delta}\right|^{2}(t)+\int_{0}^{t} \int_{\Omega} \mu\left|D\left(u_{n}-u^{\delta}\right)\right|^{2}+\xi\left|\operatorname{div}\left(u_{n}-u^{\delta}\right)\right|^{2} \leq \\
+\int_{\Omega}\left(\sqrt{\rho_{n}}-1\right) \sqrt{\rho_{n}} u_{n} \cdot u^{\delta}(t)+ \\
-\int_{0}^{t} \int_{\Omega} \rho_{n} u_{n}\left[\partial_{t} u^{\delta}+u^{\delta} \cdot \nabla u^{\delta}-\mu \Delta u^{\delta}\right]-\mu \int_{0}^{t} \int_{\Omega}\left(\rho_{n}-1\right) u_{n} \cdot \Delta u^{\delta}+ \\
+\int_{0}^{t} \int_{\Omega}\left[\left(u_{n}-u^{\delta}\right)+u^{\delta}+\left(\rho_{n}-1\right) u_{n}\right]\left[\left(u_{n}-u^{\delta}\right) \cdot \nabla u^{\delta}\right]+ \\
+\int_{\Omega} \frac{1}{2}\left|\sqrt{\rho_{n}^{0}} u_{n}^{0}-u_{0}^{\delta}\right|^{2}-\int_{\Omega}\left(\sqrt{\rho_{n}^{0}}-1\right) \sqrt{\rho_{n}^{0}} u_{n}^{0} \cdot u_{0}^{\delta}
\end{array}\right.
$$

Using the Gagliardo-Nirenberg inequality, we get for almost all $\ell$

$$
\begin{aligned}
& \left|\int_{\Omega}\left(u_{n}-u^{\delta}\right)\left[\left(u_{n}-u^{\delta}\right) \cdot \nabla u^{\delta}\right]\right| \leq \\
\leq & \left\|u_{n}-u^{\delta}\right\|_{L^{4}}^{2}\left\|\nabla u^{\delta}\right\|_{L^{2}} \\
\leq & \left\|u_{n}-u^{\delta}\right\|_{L^{2}}\left\|D\left(u_{n}-u^{\delta}\right)\right\|_{L^{2}}\left\|\nabla u^{\delta}\right\|_{L^{2}} \\
\leq & {\left[\left\|\sqrt{\rho_{n}} u_{n}-u^{\delta}\right\|_{L^{2}}+\left\|\left(\sqrt{\rho_{n}}-1\right) u_{n}\right\|_{L^{2}}\right]\left\|D\left(u_{n}-u^{\delta}\right)\right\|_{L^{2}}\left\|\nabla u^{\delta}\right\|_{L^{2}} }
\end{aligned}
$$


and then, by the Cauchy-Schwarz inequality, we get

$$
\begin{aligned}
& \left\|\sqrt{\rho_{n}} u_{n}-u^{\delta}\right\|_{L^{2}}\left\|D\left(u_{n}-u^{\delta}\right)\right\|_{L^{2}}\left\|\nabla u^{\delta}\right\|_{L^{2}} \leq \\
\leq & \frac{\nu}{2}\left\|D\left(u_{n}-u^{\delta}\right)\right\|_{L^{2}}^{2}+\frac{1}{2 \nu}\left\|\sqrt{\rho_{n}} u_{n}-u^{\delta}\right\|_{L^{2}}^{2}\left\|\nabla u^{\delta}\right\|_{L^{2}}^{2}
\end{aligned}
$$

where $\nu=\inf (\mu, \mu+\xi)$.

Next, let $A_{n}^{\delta}(t)$ be defined by

$$
(81)\left\{\begin{array}{l}
A_{n}^{\delta}(t)=\mid \int_{\Omega}\left(\sqrt{\rho_{n}}-1\right) \sqrt{\rho_{n}} u_{n} \cdot u^{\delta}(t)+ \\
-\int_{0}^{t} \int_{\Omega} \rho_{n} u_{n}\left[\partial_{t} u^{\delta}+u^{\delta} \cdot \nabla u^{\delta}-\mu \Delta u^{\delta}\right]-\mu \int_{0}^{t} \int_{\Omega}\left(\rho_{n}-1\right) u_{n} \cdot \Delta u^{\delta}+ \\
+\int_{0}^{t} \int_{\Omega}\left[u^{\delta}+\left(\rho_{n}-1\right) u_{n}\right]\left[\left(u_{n}-u^{\delta}\right) \cdot \nabla u^{\delta}\right]-\int_{\Omega}\left(\sqrt{\rho_{n}^{0}}-1\right) \sqrt{\rho_{n}^{0}} u_{n}^{0} \cdot u_{0}^{\delta}+ \\
+\left\|\left(\sqrt{\rho_{n}}-1\right) u_{n}\right\|_{L^{2}}\left\|D\left(u_{n}-u^{\delta}\right)\right\|_{L^{2}}\left\|\nabla u^{\delta}\right\|_{L^{2}} \mid
\end{array}\right.
$$

Then, we see easily that for all $\delta, A_{n}^{\delta}(t)$ converges to 0 for almost all $t$, uniformly in $t$ and that $\int A_{n}^{\delta}(t) d t$ goes to 0 when $n$ goes to $\infty$. In deed for the terms containing the factor $\left(\rho_{n}-1\right)$ or $\left(\sqrt{\rho_{n}}-1\right)$, we use that

$$
\left\|\sqrt{\rho_{n}}-1\right\|_{L^{\infty}\left(0, T ; L^{p}\right)} \leq\left\|\rho_{n}-1\right\|_{L^{\infty}\left(0, T ; L^{p}\right) \underset{n}{\rightarrow} 0}
$$

and for the two others, we use the weak convergence of $\rho_{n} u_{n}$ to $v$ which is divergence-free and the weak convergence of $u_{n}-u^{\delta}$ to $v-u^{\delta}$ and then notice that

$$
\begin{gathered}
\int_{0}^{t} \int_{\Omega} v\left[\partial_{t} u^{\delta}+u^{\delta} \cdot \nabla u^{\delta}-\mu \Delta u^{\delta}\right]=\int_{0}^{t} \int_{\Omega} v \cdot \nabla \pi=0 \\
\int_{0}^{t} \int_{\Omega} u^{\delta}\left[\left(v-u^{\delta}\right) \cdot \nabla u^{\delta}\right]=-\int_{0}^{t} \int_{\Omega} \frac{\left|u^{\delta}\right|^{2}}{2} \operatorname{div}\left(v-u^{\delta}\right)=0 .
\end{gathered}
$$

The fact that the convergence is uniform in time for these two terms can be deduced from the following elementray lemma

LEMMA 6.2. - If $f_{n}$ is bounded in $L^{\alpha}(0, T)$ with $\alpha>1$ and $f_{n}$ converges weakly to 0 , then

$$
\int_{0}^{t} f_{n} \rightarrow 0
$$

uniformly in $t$.

Vol. 16, n 3-1999. 
Hence, (80) yields for almost all $t$

$$
\left\{\begin{array}{l}
\int_{\Omega} \frac{1}{2}\left|\sqrt{\rho_{n}} u_{n}-u^{\delta}\right|^{2}(t)+\int_{0}^{t} \int_{\Omega} \frac{\nu}{2}\left|D\left(u_{n}-u^{\delta}\right)\right|^{2} \leq \\
A_{n}^{\delta}(t)+\int_{\Omega} \frac{1}{2}\left|\sqrt{\rho_{n}^{0}} u_{n}^{0}-u_{0}^{\delta}\right|^{2}+C \int_{0}^{t} \int_{\Omega}\left\|\sqrt{\rho_{n}} u_{n}-u^{\delta}\right\|_{L^{2}}^{2}\left\|\nabla u^{\delta}\right\|_{L^{2}}^{2} \cdot
\end{array}\right.
$$

By Grönwall's inequality, we deduce that we have for almost all $t \in(0, \infty)$

$$
\left\{\begin{array}{l}
\int_{\Omega} \frac{1}{2}\left|\sqrt{\rho_{n}} u_{n}-u^{\delta}\right|^{2}(t) \leq \\
\leq\left[\int_{\Omega} \frac{1}{2}\left|\sqrt{\rho_{n}^{0}} u_{n}^{0}-u_{0}^{\delta}\right|^{2}+\sup _{0 \leq s \leq T} A_{n}^{\delta}(s)\right] e^{C \int_{0}^{t}\left\|\nabla u^{\delta}(s)\right\|_{L^{2}}^{2}}
\end{array}\right.
$$

Then, letting $n$ go to infinity, we obtain

$$
\begin{aligned}
\left\|v-u^{\delta}(t)\right\|_{L^{\infty}\left(0, T ; L^{2}(\Omega)\right.} & \leq \varlimsup_{n}\left\|\sqrt{\rho_{n}} u_{n}-u^{\delta}\right\|_{L^{\infty}\left(0, T ; L^{2}(\Omega)\right.} \\
& \leq C_{0}\left\|u^{0}-u_{0}^{\delta}\right\|_{L^{2}(\Omega)}
\end{aligned}
$$

where

$$
C_{0}=\sup _{0<\delta<1} e^{C \int_{0}^{T}\left\|\nabla u^{\delta}(s)\right\|_{L^{2}}^{2}}<+\infty .
$$

Then, letting $\delta$ go to infinity, we recover the uniform convergence in $t$ of $\sqrt{\rho_{n}} u_{n}$ to $u$, since

$$
\begin{aligned}
& \varlimsup_{n}\left\|\sqrt{\rho_{n}} u_{n}-u\right\|_{L^{\infty}\left(0, T ; L^{2}\right)} \\
& \quad \leq \lim _{\delta}\left[C_{0}\left\|u^{0}-u_{0}^{\delta}\right\|_{L^{2}(\Omega)}+\left\|u^{\delta}-u\right\|_{L^{\infty}\left(0, T ; L^{2}\right)}\right]=0
\end{aligned}
$$

Going back to (82), we get that $D u_{n}$ converges in $L^{2}$ to $D u$ and that $u_{n}$ converges to $u$ in $L^{2}\left(0, T ; H^{1}(\Omega)\right.$. Finally, we also get that $\nabla \pi_{n}$ converges weakly to $\nabla \pi$ in $H^{-1}((0, T) \times \Omega)$ for instance.

Remark. - It is worth noticing that the above proof shows that, for $N \geq 2$, the result is still true on any interval of time $(0, T)$ for which we know that there exists a solution $u$ (which is in fact unique) of the incompressible Navier-Stokes equations corresponding to $u^{0}$ which satisfies :

$$
D u \in L^{1}\left(0, T ; L^{\infty}(\Omega)\right)+L^{2}\left(0, T ; L^{N}(\Omega)\right) .
$$


The second issue, we are going to study concerns the convergence of solutions of the compressible Navier-Stokes system (54) as $\gamma_{n}$ goes to infinity in the case where $f \rho_{n}^{0}=M \geq 1$. Let $\left(\rho_{n}, u_{n}\right)$ be a sequence of solutions of (54) satisfying in addition (55) and (56) and such that $f \rho_{n}^{0}=M \geq 1, f\left(\rho_{n}^{0}\right)^{\gamma_{n}} \leq M^{\gamma_{n}}+C_{n} \gamma_{n}$ for some constant $C_{n}$, such that $C_{n}$ converges to 0 and $\sqrt{\rho_{n}^{0}} u_{n}^{0}$ converges strongly in $L^{2}$ to a divergence-free vector $u^{0}$.

THEOREM 6.3. - Under the above assumptions, $\rho_{n}$ converges to $M$ in $C\left([0, T] ; L^{p}(\Omega)\right)$ for $1 \leq p<+\infty, \sqrt{\rho_{n}} u_{n}$ converges to $u$ in $L^{\infty}\left(0, T ; L^{2}(\Omega)\right)$ and $D u_{n}$ converges to $D u$ in $L^{2}\left(0, T ; L^{2}(\Omega)\right)$ for all $T \in(0, \infty)$ where $u$ is the unique solution of the incompressible NavierStokes equations corresponding to the initial condition $u^{0}$ and where the viscosity $\mu$ is replaced by $\mu / M$.

Proof. - The proof of this theorem is the same as the previous one. The only point we must show is how we obtain uniform bounds. In fact the energy inequality (56) reads for almost all $t$

$$
\left\{\begin{array}{l}
\int \frac{1}{2} \rho_{n}\left|u_{n}\right|^{2}(t)+\frac{a}{\gamma_{n}-1}\left(\rho_{n}\right)^{\gamma_{n}}(t)+\int_{0}^{t} \int \mu\left|D u_{n}\right|^{2}+\xi\left(\text { divu }_{n}\right)^{2} \leq \\
\leq \int \frac{1}{2} \rho_{n}^{0}\left|u_{n}^{0}\right|^{2}+\frac{a}{\gamma_{n}-1}\left(\rho_{n}^{0}\right)^{\gamma_{n}} \leq \int \frac{1}{2} \rho_{n}^{0}\left|u_{n}^{0}\right|^{2}+\frac{a}{\gamma_{n}-1}|\Omega| M^{\gamma_{n}}+C
\end{array}\right.
$$

Hence, to get uniform bounds, we notice that we deduce from Jensen's inequality that we have for almost all $t$

$$
\int_{\Omega}\left(\rho_{n}\right)^{\gamma_{n}}(t) \geq|\Omega|\left[\int_{\Omega} \rho_{n}\right]^{\gamma_{n}}(t)=|\Omega| M^{\gamma_{n}} .
$$

Arguing as above, we can extract subsequences that converge weakly. Next, to see that $\rho \equiv M$ and that $\rho_{n}$ converges strongly to $M$ we use that for all $p(1<p<+\infty)$ and for $n$ large enough $\left(\gamma_{n}>p\right)$, we have

$$
\begin{aligned}
\left\|\rho_{n}\right\|_{L^{\infty}\left(0, T ; L^{p}\right)} & \leq\left\|\rho_{n}\right\|_{L^{\infty}\left(0, T ; L^{1}\right)}^{\theta_{n}}\left\|\rho_{n}\right\|_{L^{\infty}\left(0, T ; L^{\gamma_{n}}\right)}^{1-\theta_{n}} \\
& \leq M_{n}^{\theta_{n}}\left(C \gamma_{n}+|\Omega| M^{\gamma_{n}}\right)^{\left(1-\theta_{n}\right) / \gamma_{n}}
\end{aligned}
$$

where $\theta_{n}$ is given for any $n$ by $\frac{1}{p}=\theta_{n}+\frac{1-\theta_{n}}{\gamma_{n}}$. Then, letting $n$ go to infinity, we deduce that

$$
\|\rho\|_{L^{\infty}\left(0, T ; L^{p}\right)} \leq \liminf _{n \rightarrow \infty}\left\|\rho_{n}\right\|_{L^{\infty}\left(0, T ; L^{p}\right)} \leq M
$$

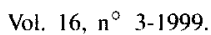


Then, since $f \rho(t)=M$ for all $t$, we get, using the Jensen inequality (or letting $p$ go to infinity) that $\rho \equiv M$. We also deduce the convergence of $\rho_{n}$ to $M$ in $L^{p}\left(0, T ; L^{p}\right)$. Next, to get the convergence in $L^{\infty}\left(0, T ; L^{p}\right)$, we write that

$$
\rho_{n}^{\gamma_{n}}>M^{\gamma_{n}}\left[1+\gamma_{n} \frac{\left(\rho_{n}-M\right)_{+}}{M}+\frac{\gamma_{n}\left(\gamma_{n}-1\right)}{2}\left(\frac{\left(\rho_{n}-M\right)_{+}}{M}\right)^{2}\right]
$$

and deduce easily from the bounds we have on $\rho_{n}^{\gamma_{n}}$ that $\left(\rho_{n}-M\right)_{+}$ converges to 0 in $L^{\infty}\left(0, T ; L^{2}\right)$ and then in $L^{\infty}\left(0, T ; L^{1}\right)$. Using that $f \rho_{n}=M$, we get that $(\rho-M)_{-}$goes to 0 in $L^{\infty}\left(0, T ; L^{1}\right)$. Finally, to conclude we remark that we can use the expansion of $\rho_{\eta \prime}^{\gamma}$ up to the order $p$ as in (85) and deduce that $\left(\rho_{n}-M\right)_{+}$tends to 0 in $L^{\infty}\left(0, T ; L^{p}\right)$. Then, we see that

$$
\int\left|\rho_{n}-M\right|^{p} \leq \int\left(\rho_{n}-M\right)_{+}^{p}+M^{p-1}\left(M-\rho_{n}\right)_{+} .
$$

And letting $n$ go to infinity, we conclude easily.

Eventually, we see that the proof of the previous theorem can be adapted to this case. The study of more general initial data will be considered in a forthcoming paper.

\section{REFERENCES}

[1] Bajsanski and R. Colfman, in Proc. Symp. Pure Math., 10, AMS.

[2] R. COIFMAN and Y. MEYER, On commutators of singular integrals and bilinear singular integrals., Trans. Amer. Math. Soc., Vol. 212, 1975, pp. 315-331.

[3] R. CoIfman, R. Rochberg and G. Weiss, Factorization theorems for Hardy spaces in several variables, Ann. of Math., Vol. 2, 103, 1976, no. 3, pp. 611-635.

[4] J. LERAY, Étude de diverses équations intégrales nonlinéaires et de quelques problèmes que pose l'hydrodynamique, J. Math. Pure Appl., Vol. 12, 1933, pp. 1-82.

[5] J. LERAY, Essais sur le mouvement d'un liquide visqueux emplissant l'espace, Acta Math., Vol. 63, 1934, pp. 193-248.

[6] P.-L. Lions, Mathematical Topics in Fluid Mechanics. Vol. 2, Compressible Models, Clarendon Press, Oxford Science Publications, Oxford, 1998.

[7] P.-L. Lions, Mathematical Topics in Fluid Mechanics. Vol. 1, Incompressible Models, Clarendon Press, Oxford Science Publications, Oxford, 1996.

[8] P.-L. Lions, Existence globale de solutions pour les équations de Navier-Stokes compressibles isentropiques, C. R. Acad. Sci. Paris, Vol. 316, 1993, pp. 1335-1340.

[9] P.-L. Lons, Compacité des solutions des équations de Navier-Stokes compressibles isentropiques, C. R. Acad. Sci. Paris, Vol. 317, 1993, pp. 115-120.

[10] P.-L. Lions, Limites incompressible et acoustique pour des fluides visqueux, compressibles et isentropiques, C. R. Acad. Sci. Paris, Vol. 317, 1993, pp. 1197-1202.

[11] P.-L. Lions and N. Masmoudi, Incompressible limit of a viscous compressible fluid, to appear in J. Math. Pure Appl., Vol. 77, 1998, pp. 585-627.

(Manuscript received April 8. 1997.) 